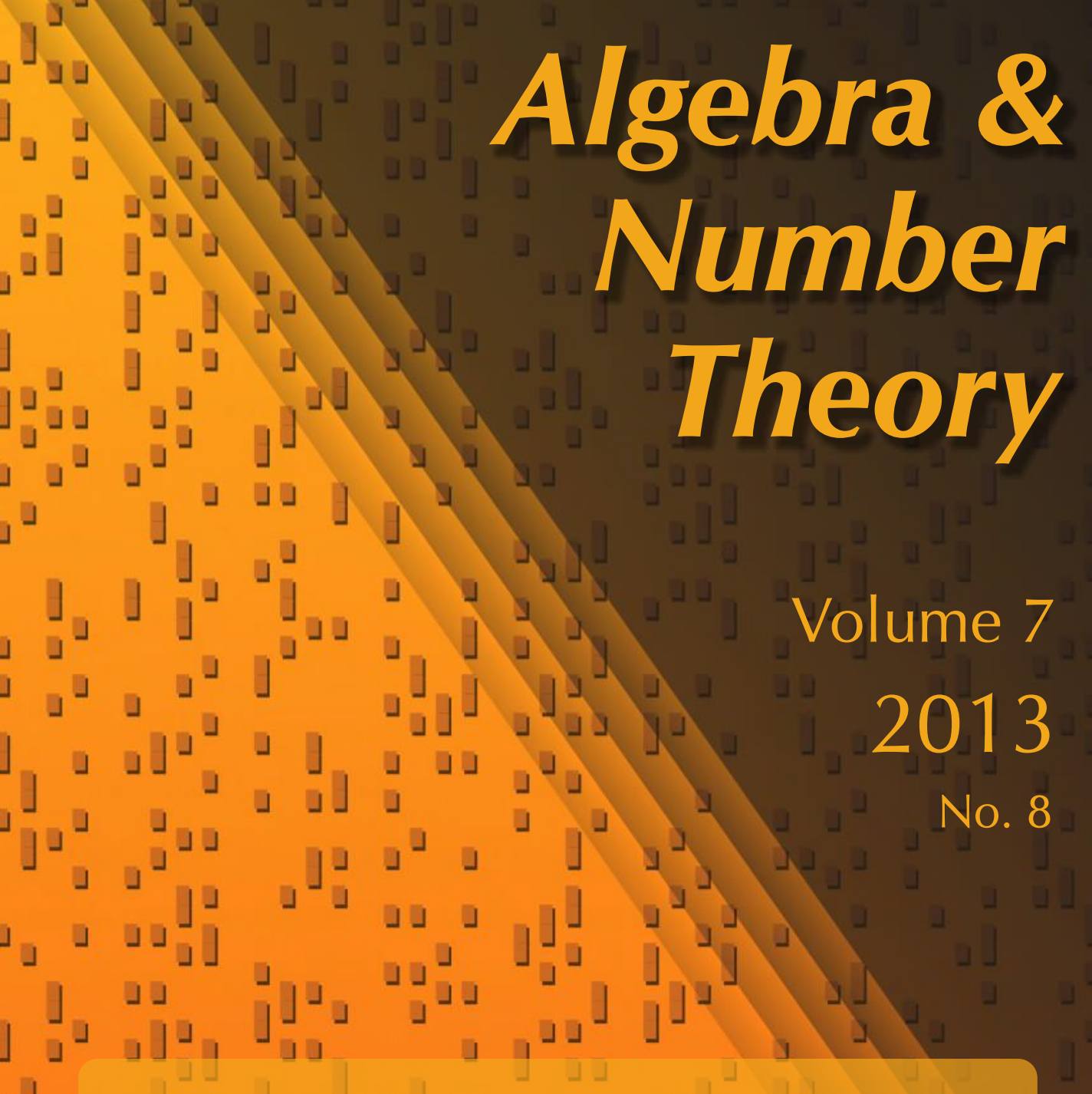

Division algebras and quadratic forms over fraction fields of two-dimensional henselian domains

Yong $\mathrm{Hu}$

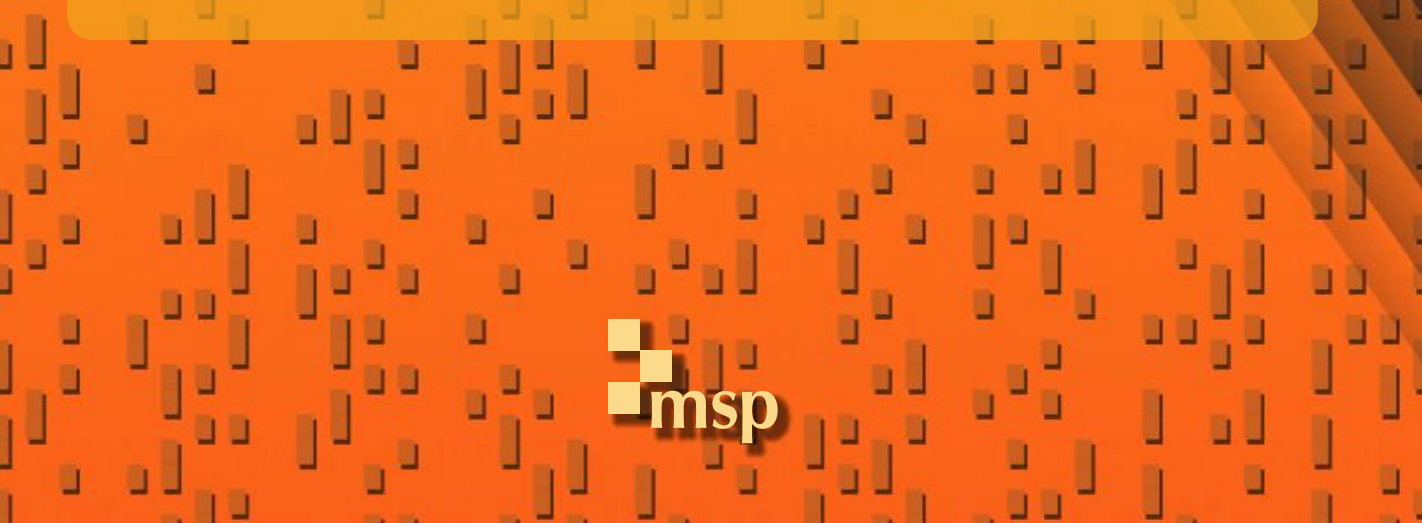




\title{
Division algebras and quadratic forms over fraction fields of two-dimensional henselian domains
}

\author{
Yong $\mathrm{Hu}$
}

Let $K$ be the fraction field of a two-dimensional, henselian, excellent local domain with finite residue field $k$. When the characteristic of $k$ is not 2 , we prove that every quadratic form of rank $\geq 9$ is isotropic over $K$ using methods of Parimala and Suresh, and we obtain the local-global principle for isotropy of quadratic forms of rank 5 with respect to discrete valuations of $K$. The latter result is proved by making a careful study of ramification and cyclicity of division algebras over the field $K$, following Saltman's methods. A key step is the proof of the following result, which answers a question of Colliot-Thélène, Ojanguren and Parimala: for a Brauer class over $K$ of prime order $q$ different from the characteristic of $k$, if it is cyclic of degree $q$ over the completed field $K_{v}$ for every discrete valuation $v$ of $K$, then the same holds over $K$. This local-global principle for cyclicity is also established over function fields of $p$-adic curves with the same method.

\section{Introduction}

Division algebras and quadratic forms over a field have been objects of interest in classical and modern theories of algebra and number theory. They may also be naturally and closely related to the study of semisimple algebraic groups of classical types. In recent years, there has been much interest in problems on division algebras and quadratic forms over function fields of two-dimensional integral schemes (which we call surfaces).

Mostly, surfaces that have been studied are those equipped with a dominant quasiprojective morphism to the spectrum of a normal, henselian, excellent local domain $A$. If $A$ is of (Krull) dimension 0, these are algebraic surfaces over a field. Over function fields of these surfaces, de Jong [2004] and Lieblich [2011b] have proven remarkable theorems concerning the period-index problem. If $A$ is of dimension 1, the surfaces of interest are called arithmetic surfaces by some authors. Over function fields of arithmetic surfaces, several methods have been developed to study division algebras and/or quadratic forms, for example in [Saltman 1997;

MSC2010: primary 11E04; secondary 16K99.

Keywords: quadratic forms, division algebras, local-global principle. 
2007; 2008; Lieblich 2011a; Harbater et al. 2009]. The methods pioneered in the series of papers by Saltman have been important ingredients in several works by others, including the proof of Parimala and Suresh $[2010 ; 2012]$ of the fact that over a nondyadic $p$-adic function field every quadratic form of dimension $\geq 9$ has a nontrivial zero. In contrast with the arithmetic case, it seems that in the case where $A$ is two-dimensional, fewer results have been established in earlier work.

In this paper, we concentrate on the study of division algebras and quadratic forms over the function field $K$ of a surface that admits a proper birational morphism to the spectrum of a two-dimensional, henselian, excellent local domain $R$. The spectrum Spec $R$ will sometimes be called a local henselian surface, and a regular surface $X$ equipped with a proper birational morphism $X \rightarrow \operatorname{Spec} R$ will be referred to as a regular proper model of Spec $R$. As typical examples, one may take $R$ to be the henselization at a closed point of an algebraic or an arithmetic surface or the integral closure of the ring $A \llbracket t \rrbracket$ of formal power series in a finite extension of its fraction field Frac $(A \llbracket t \rrbracket)$, where $A$ is a complete discrete valuation ring. Note that the ring $R$ need not be regular in our context.

Let $k$ denote the residue field of $R$. When $k$ is separably closed, many problems over the function field $K$ (e.g., period-index, cyclicity of division algebras, $u$ invariant and local-global principle for quadratic forms of lower dimension) have been solved by Colliot-Thélène, Ojanguren and Parimala [Colliot-Thélène et al. 2002]. In the case with $k$ finite, only the local-global principle for quadratic forms of rank 3 or 4 is proved in that paper. Harbater, Hartmann, and Krashen [Harbater et al. 2011] obtained some results with less restrictive assumptions on the residue field but more restrictions on the shape of the ring $R$.

While the proofs pass through many analyses on ramification of division algebras, our primary goals are the following two theorems on quadratic forms:

Theorem 1.1. Let $R$ be a two-dimensional, henselian, excellent local domain with finite residue field $k$ and fraction field $K$. Assume that 2 is invertible in $k$. Let $\Omega_{R}$ be the set of discrete valuations of $K$ that correspond to codimension-1 points of regular proper models of Spec $R$.

Then quadratic forms of rank 5 over $K$ satisfy the local-global principle with respect to discrete valuations in $\Omega_{R}$; namely, if a quadratic form $\phi$ of rank 5 over $K$ has a nontrivial zero over the completed field $K_{v}$ for every $v \in \Omega_{R}$, then $\phi$ has nontrivial zero over $K$.

The next theorem amounts to saying that the field $K$ has $u$-invariant (page 1945) equal to 8:

Theorem 1.2. Let $R$ be a two-dimensional, henselian, excellent local domain with finite residue field $k$ and fraction field $K$. Assume that 2 is invertible in $k$.

Then every quadratic form of rank $\geq 9$ has a nontrivial zero over $K$. 
Over the function field of an arithmetic surface over a complete discrete valuation ring, the same local-global principle as in Theorem 1.1 is proved for all quadratic forms of rank $\geq 3$ in [Colliot-Thélène et al. 2012, Theorem 3.1] by using the patching method of [Harbater et al. 2009]. In the case that $R=A \llbracket t \rrbracket$ is a ring of formal power series in one variable over a complete discrete valuation ring $A$, the same type of local-global principle has been proven for quadratic forms of rank $\geq 5$ in [Hu 2012b] using the arithmetic case established by Colliot-Thélène, Parimala and Suresh [Colliot-Thélène et al. 2012]. (See Remark 4.3 for more information.) However, in the general local henselian case, the lack of an appropriate patching method has been an obstacle to proving the parallel local-global result. So for a field $K$ as in Theorem 1.1, the local-global principle for quadratic forms of rank 6 , 7 or 8 remains open.

In the case of a $p$-adic function field, it is known that at least three methods can be used to determine the $u$-invariant: the cohomological method of [Parimala and Suresh 2010], the patching method of [Harbater et al. 2009] and the method of [Leep 2013], which is built on results from [Heath-Brown 2010]. But in the case of the function field of a local henselian surface considered here, not all of them seem to still work. For the fraction field of a power series ring $R=A \llbracket t \rrbracket$ over a complete discrete valuation ring with finite residue field, it is known that the $u$-invariant is at most 8 [Harbater et al. 2009, Corollary 4.19]. Our proof of this result for general $R$ (with finite residue field) follows the method of Parimala and Suresh [2010; 2012].

Theorem 1.2 implies that the $u$-invariants $u(K)$ of the fraction field $K$ and $u(k)$ of the residue field $k$ satisfy the relation $u(K)=4 u(k)$ when the residue field $k$ is finite. A question of Suresh asks if this relation still holds when $k$ is an arbitrary field of characteristic $\neq 2$. The answer is known to be affirmative in some other special cases, but the general case seems to remain open. (See Question 4.8 for more information.)

As a byproduct, we also obtain (under the assumption of Theorem 1.2) a localglobal principle for torsors of the special orthogonal group $\mathrm{SO}(\phi)$ of a quadratic form $\phi$ of rank $\geq 2$ over $K$ (Theorem 4.9). In fact, Theorem 1.2 will also be useful in the study of local-global principle for torsors under some simply connected groups of classical types over $K$ [Hu 2012a].

The main tools we will need to prove Theorem 1.1 come from technical analyses of ramification behaviors of division algebras using methods developed by Saltman [1997; 2007; 2008]. A key ingredient is the following result:

Theorem 1.3. Let $R$ be a two-dimensional, henselian, excellent local domain with finite residue field $k, q$ a prime number unequal to the characteristic of $k, K$ the fraction field of $R$ and $\alpha \in \operatorname{Br}(K)$ a Brauer class of order $q$. Let $\Omega_{R}$ be the set of discrete valuations of $K$ that correspond to codimension-1 points of regular proper models of Spec $R$. 
If for every $v \in \Omega_{R}$, the Brauer class

$$
\alpha \otimes_{K} K_{v} \in \operatorname{Br}\left(K_{v}\right)
$$

is represented by a cyclic algebra of degree $q$ over the completed field $K_{v}$, then $\alpha$ is represented by a cyclic algebra of degree $q$ over $K$.

Actually, as the same proof applies to the function field of a $p$-adic curve, a similar result over $p$-adic function fields, which seems not to have been treated in the literature, holds as well (Theorem 3.21). Note that a special case of Theorem 1.3 answers a question raised in [Colliot-Thélène et al. 2002, Remark 3.7].

Here is a brief description of the organization of the paper, together with some auxiliary results obtained in the process of proving the above-mentioned theorems.

Section 2 is concerned with preliminary reviews on Brauer groups and Galois symbols. The goal is to introduce some basic notions and recall standard results that we will frequently use later. In Section 3, we recall some of the most useful techniques and results from Saltman's papers and we prove Theorem 1.3. We also prove over the field $K$ considered in Theorem 1.1 two local analogs of more global results Saltman had shown: that the index of a Brauer class of period prime to the residue characteristic divides the square of its period and that a class of prime index $q$ that is different from the residue characteristic is represented by a cyclic algebra of degree $q$. This last statement is proved by generalizing a result of Saltman on modified Picard groups. Finally, we will concentrate on results about quadratic forms in Section 4. The proofs of Theorems 1.1 and 1.2 build upon the work of Parimala and Suresh and on a result from Saito's class field theory for two-dimensional local rings [Saito 1987].

To ease the discussions, we fix some notations and terminological conventions for all the rest of the paper.

- All schemes are assumed to be noetherian and separated. All rings under consideration will be noetherian (commutative with 1).

- A curve or surface means an integral scheme of dimension 1 or 2, respectively.

- Given a scheme $X$, we denote by $\operatorname{Br}(X)$ its cohomological Brauer group, i.e., $\operatorname{Br}(X):=H_{\text {êt }}^{2}\left(X, \mathbb{G}_{m}\right)$. If $X=\operatorname{Spec} A$ is affine, we write $\operatorname{Br}(A)$ instead of $\operatorname{Br}(\operatorname{Spec} A)$.

- If $X$ is a scheme and $x \in X$, we write $\kappa(x)$ for the residue field of $x$, and if $Z \subseteq X$ is an irreducible closed subset with generic point $\eta$, then we write $\kappa(Z):=\kappa(\eta)$.

- The reduced closed subscheme of a scheme $X$ will be written as $X_{\text {red }}$.

- A discrete valuation will always be assumed normalized (nontrivial) and of rank 1. 
- Given a field $F$ and a scheme $X$ together with a morphism Spec $F \rightarrow X$, $\Omega(F / X)$ will denote the set of discrete valuations of $F$ that have a center on $X$. If $X=\operatorname{Spec} A$ is affine, we write $\Omega(F / A)$ instead of $\Omega(F / \operatorname{Spec} A)$.

- Given a scheme $X$ and $i \in \mathbb{N}$, we denote by $X^{(i)}$ the set of codimension- $i$ points of $X$, i.e., $X^{(i)}:=\left\{x \in X \mid \operatorname{dim} 0_{X, x}=i\right\}$. If $X$ is a normal integral scheme with function field $F$, we will sometimes identify $X^{(1)}$ with the set of discrete valuations of $F$ corresponding to points in $X^{(1)}$.

- For an abelian group $A$ and a positive integer $n$, let $A[n]$ denote the subgroup consisting of $n$-torsion elements of $A$ and let $A / n=A / n A$ so that there is a natural exact sequence

$$
0 \rightarrow A[n] \rightarrow A \stackrel{n}{\rightarrow} A \rightarrow A / n \rightarrow 0 .
$$

- Given a field $F$, let $F_{S}$ be a fixed separable closure of $F$ and $G_{F}:=\operatorname{Gal}\left(F_{S} / F\right)$ the absolute Galois group. Galois cohomology $H^{i}\left(G_{F}, \cdot\right)$ of the group $G_{F}$ will be written $H^{i}(F, \cdot)$ instead.

- $R$ will always denote a two-dimensional, henselian, excellent local domain with fraction field $K$ and residue field $k$.

- By a regular proper model of $\operatorname{Spec} R$, we mean a regular integral scheme $\mathscr{X}$ equipped with a proper birational morphism $\mathscr{X} \rightarrow \operatorname{Spec} R$. A discrete valuation of $K$ that corresponds to a codimension-1 point of a regular proper model of Spec $R$ will be referred to as a divisorial valuation of $K$. We denote by $\Omega_{R}$ the set of divisorial valuations of $K$.

\section{Some preliminaries}

Brauer groups of low-dimensional schemes. Since we will often use arguments related to Brauer groups of curves or surfaces, let us briefly review some basic facts in this respect.

Theorem 2.1 [Grothendieck 1968a; Colliot-Thélène et al. 2002]. Let X be a (noetherian) scheme of dimension d.

(i) If $d \leq 1$, then the natural map $\operatorname{Br}(X) \rightarrow \operatorname{Br}\left(X_{\text {red }}\right)$ is an isomorphism.

(ii) If $X$ is regular and integral with function field $F$, then the natural map $\operatorname{Br}(X) \rightarrow \operatorname{Br}(F)$ is injective.

(iii) If $X$ is regular, integral with function field $F$ and of dimension $d \leq 2$, then $\operatorname{Br}(X)=\bigcap_{x \in X^{(1)}} \operatorname{Br}\left(\mathscr{O}_{X, x}\right)$ inside $\operatorname{Br}(F)$.

(iv) Let $A$ be a henselian local ring, and let $X \rightarrow \operatorname{Spec} A$ be a proper morphism whose closed fiber $X_{0}$ has dimension $\leq 1$. If $X$ is regular and of dimension 2, then the natural map $\operatorname{Br}(X) \rightarrow \operatorname{Br}\left(X_{0}\right)$ is an isomorphism. 
Proof. See [Colliot-Thélène et al. 2002, Lemma 1.6] for (i), [Grothendieck 1968a, Corollary 1.8] for (ii), [Grothendieck 1968a, Corollary 2.2, Proposition 2.3] for (iii) and [Colliot-Thélène et al. 2002, Theorem 1.8(c)] for (iv).

The following property for fields, already considered in [Saltman 1997], will be of interest to us:

Definition 2.2. We say a field $k$ has property $B_{1}$ or $k$ is a $B_{1}$ field if, for every proper regular integral (not necessarily geometrically integral) curve $C$ over the field $k$, one has $\operatorname{Br}(C)=0$.

Example 2.3. Here are some examples of $B_{1}$ fields:

(1) A separably closed field $k$ has property $B_{1}$ [Grothendieck 1968b, Corollary 5.8].

(2) A finite field $k$ has property $B_{1}$. This is classical by class field theory; see also [Grothendieck 1968b, p. 97].

(3) If $k$ has property $B_{1}$, then so does any algebraic field extension $k^{\prime}$ of $k$.

Proposition 2.4. Let $k$ be a $B_{1}$ field.

(i) For any proper $k$-scheme $X$ of dimension $\leq 1$, one has $\operatorname{Br}(X)=0$.

(ii) The cohomological dimension $\operatorname{cd}(k)$ of $k$ is $\leq 1$; i.e., for every torsion $G_{k^{-}}$ module $A, H^{i}(k, A)=0$ for all $i \geq 2$.

(iii) If the characteristic of $k$ is not 2 , then every quadratic form of rank $\geq 3$ has a nontrivial zero over $k$.

Proof. (i) By Theorem 2.1(i), we may assume $X$ is reduced.

For the zero-dimensional case, it suffices to prove that $\operatorname{Br}(L)=0$ for a finite extension field $L$ of $k$. Indeed, the $B_{1}$ property implies that $\operatorname{Br}\left(\mathbb{P}_{L}^{1}\right)=0$. The existence of $L$-rational points on $\mathbb{P}_{L}^{1}$ shows that the natural map $\operatorname{Br}(L) \rightarrow \operatorname{Br}\left(\mathbb{P}_{L}^{1}\right)$ induced by the structural morphism $\mathbb{P}_{L}^{1} \rightarrow \operatorname{Spec} L$ is injective. Hence, $\operatorname{Br}(L)=0$.

Now assume that $X$ is reduced of dimension 1. Let $X^{\prime} \rightarrow X$ be the normalization of $X$. By [Colliot-Thélène et al. 2002, Proposition 1.14], there is a zero-dimensional closed subscheme $D$ of $X$ such that the natural map $\operatorname{Br}(X) \rightarrow \operatorname{Br}\left(X^{\prime}\right) \times \operatorname{Br}(D)$ is injective. Now $X^{\prime}$ is a disjoint union of finitely many proper regular $k$-curves, so $\operatorname{Br}\left(X^{\prime}\right)=0$ by the $B_{1}$ property. We have $\operatorname{Br}(D)=0$ by the zero-dimensional case, whence $\operatorname{Br}(X)=0$ as desired.

(ii) As a special case of (i), we have $\operatorname{Br}\left(k^{\prime}\right)=0$ for every finite separable extension field $k^{\prime}$ of $k$. This implies $\operatorname{cd}(k) \leq 1$ by [Serre 1994, p. 88, Proposition 5].

(iii) By (ii), we have in particular $\operatorname{Br}(k)[2]=H^{2}\left(k, \mu_{2}\right)=0$. Thus, every quaternion algebra over $k$ is split and the associated quadric has a $k$-rational point. Up to a scalar multiple, every nonsingular three-dimensional quadratic form is associated to a quaternion algebra and hence isotropic. 
The following corollary is essentially proven in [Colliot-Thélène et al. 2002, Corollaries 1.10 and 1.11]:

Corollary 2.5. Let $A$ be a (noetherian) henselian local ring, and let $X \rightarrow \operatorname{Spec} A$ be a proper morphism whose closed fiber $X_{0}$ is of dimension $\leq 1$. Assume that the residue field of $A$ has property $B_{1}$.

If $X$ is regular and of dimension 2, then $\operatorname{Br}(X)=0$.

Proof. Combine Theorem 2.1(iv) and Proposition 2.4(i).

Symbols and unramified cohomology. This subsection is devoted to a quick review of a few standard facts about Galois symbols and residue maps. For more information, we refer the reader to [Colliot-Thélène 1995].

Let $F$ be a field and $v$ a discrete valuation of $F$ with valuation ring $O_{v}$ and residue field $\kappa(v)$. Let $n>0$ be a positive integer unequal to the characteristic of $\kappa(v)$. Let $\mu_{n}$ be the Galois module on the group of $n$-th roots of unity. For an integer $j \geq 1$, let $\mu_{n}^{\otimes j}$ denote the Galois module given by the tensor product of $j$ copies of $\mu_{n}$, and define

$$
\mu_{n}^{\otimes 0}:=\mathbb{Z} / n \quad \text { and } \quad \mu_{n}^{\otimes(-j)}:=\operatorname{Hom}\left(\mu_{n}^{\otimes j}, \mathbb{Z} / n\right),
$$

where as usual $\mathbb{Z} / n$ is regarded as a trivial Galois module. Kummer theory gives a canonical isomorphism $H^{1}\left(F, \mu_{n}\right) \cong F^{*} / F^{* n}$. For an element $a \in F^{*}$, we denote by (a) its canonical image in $H^{1}\left(F, \mu_{n}\right)=F^{*} / F^{* n}$. For $\alpha \in H^{i}\left(F, \mu_{n}^{\otimes j}\right)$, the cup product $\alpha \cup(a) \in H^{i+1}\left(F, \mu_{n}^{\otimes(j+1)}\right)$ will be simply written as $(\alpha, a)$. In particular, if $a_{1}, \ldots, a_{i} \in F^{*},\left(a_{1}, \ldots, a_{i}\right) \in H^{i}\left(F, \mu_{n}^{\otimes i}\right)$ will denote the cup product $\left(a_{1}\right) \cup \cdots \cup\left(a_{i}\right) \in H^{i}\left(F, \mu_{n}^{\otimes i}\right)$. Such a cohomology class is called a symbol class.

By standard theories from Galois or étale cohomology, there are residue homomorphisms for all $i \geq 1$ and all $j \in \mathbb{Z}$

$$
\partial_{v}^{i, j}: H^{i}\left(F, \mu_{n}^{\otimes j}\right) \rightarrow H^{i-1}\left(\kappa(v), \mu_{n}^{\otimes(j-1)}\right)
$$

that fit into a long exact sequence

$$
\begin{aligned}
\cdots \rightarrow H_{\mathrm{et}}^{i}\left(\mathscr{O}_{v}, \mu_{n}^{\otimes j}\right) \rightarrow H^{i}\left(F, \mu_{n}^{\otimes j}\right) & \\
\qquad & \stackrel{\partial_{v}^{i, j}}{\longrightarrow} H^{i-1}\left(\kappa(v), \mu_{n}^{\otimes(j-1)}\right) \rightarrow H_{\text {ét }}^{i+1}\left(\mathcal{O}_{v}, \mu_{n}^{\otimes j}\right) \rightarrow \cdots .
\end{aligned}
$$

An element $\alpha \in H^{i}\left(F, \mu_{n}^{\otimes j}\right)$ is called unramified at $v$ if $\partial_{v}^{i, j}(\alpha)=0$.

Now consider the case of Brauer groups. By Theorem 2.1(ii), $\operatorname{Br}\left(\mathscr{O}_{v}\right)$ gets identified with a subgroup of $\operatorname{Br}(F)$. An element $\alpha \in \operatorname{Br}(F)$ is called unramified at $v$ if it lies in the subgroup $\operatorname{Br}\left(O_{v}\right) \subseteq \operatorname{Br}(F)$. If $n>0$ is a positive integer that is invertible in $\kappa(v)$, then an element $\alpha \in \operatorname{Br}(F)[n]$ is unramified at $v$ if and only if $\partial_{v}(\alpha)=0$, where $\partial_{v}$ denotes the residue map

$$
\partial_{v}=\partial_{v}^{2,1}: \operatorname{Br}(F)[n]=H^{2}\left(F, \mu_{n}\right) \rightarrow H^{1}(\kappa(v), \mathbb{Z} / n) .
$$


As we will frequently speak of ramification of division algebras, the above residue map $\partial_{v}=\partial_{v}^{2,1}$ will often be called the ramification map and denoted by $\operatorname{ram}_{v}$.

Let $X$ be a scheme equipped with a morphism $\operatorname{Spec} F \rightarrow X$. The subgroup

$$
\operatorname{Br}_{\mathrm{nr}}(F / X):=\bigcap_{v \in \Omega(F / X)} \operatorname{Br}\left(O_{v}\right) \subseteq \operatorname{Br}(F)
$$

where $\Omega(F / X)$ denotes the set of discrete valuations of $K$ that have a center on $X$, is referred to as the (relative) unramified Brauer group of $F$ over $X$. A Brauer class $\alpha \in \operatorname{Br}(F)$ is called unramified over $X$ if it lies in the subgroup $\operatorname{Br}_{\mathrm{nr}}(F / X)$. We say a field extension $M / F$ splits all ramification of $\alpha \in \operatorname{Br}(F)$ over $X$ if $\alpha_{M} \in \operatorname{Br}(M)$ is unramified over $X$. When $X=\operatorname{Spec} A$ is affine, we write $\operatorname{Br}_{\mathrm{nr}}(F / A)$ instead of $\operatorname{Br}_{\mathrm{nr}}(F / \operatorname{Spec} A)$.

If $X$ is an integral scheme with function field $F$ and if $X \rightarrow Y$ is a proper morphism, then $\Omega(F / X)=\Omega(F / Y)$ and hence $\mathrm{Br}_{\mathrm{nr}}(F / X)=\mathrm{Br}_{\mathrm{nr}}(F / Y)$. If $X$ is a regular curve or surface with function field $F$, then Theorem 2.1 implies that $\mathrm{Br}_{\mathrm{nr}}(F / X) \subseteq \operatorname{Br}(X)$.

Note that for any field $\kappa$, the Galois cohomology group $H^{1}(\kappa, \mathbb{Q} / \mathbb{Z})$ is identified with the group of characters of the absolute Galois group $G_{\kappa}$, i.e., the group $\operatorname{Hom}_{\mathrm{cts}}\left(G_{\kappa}, \mathbb{Q} / \mathbb{Z}\right)$ of continuous homomorphisms $f: G_{\kappa} \rightarrow \mathbb{Q} / \mathbb{Z}$. Any character $f \in \operatorname{Hom}_{\text {cts }}\left(G_{\kappa}, \mathbb{Q} / \mathbb{Z}\right)$ must have image of the form $\mathbb{Z} / m \subseteq \mathbb{Q} / \mathbb{Z}$ for some positive integer $m$, and its kernel is equal to $G_{\kappa^{\prime}}$ for some cyclic Galois extension $\kappa^{\prime} / \kappa$ of degree $m$. There is a generator $\sigma \in \operatorname{Gal}\left(\kappa^{\prime} / \kappa\right)$ such that $f(\sigma)=1+m \mathbb{Z} \in \mathbb{Z} / m$. The function $f \in \operatorname{Hom}_{\mathrm{cts}}\left(G_{\kappa}, \mathbb{Q} / \mathbb{Z}\right)$ is uniquely determined by the pair $\left(\kappa^{\prime} / \kappa, \sigma\right)$. In this paper, we will often write an element of $H^{1}(\kappa, \mathbb{Q} / \mathbb{Z})$ in this way. In particular, the ramification $\operatorname{ram}_{v}(\alpha) \in H^{1}(\kappa(v), \mathbb{Z} / n)$ of a Brauer class $\alpha \in \operatorname{Br}(F)[n]$ at a discrete valuation $v \in \Omega(F / X)$ will be represented in this way.

Let $\chi \in H^{1}(F, \mathbb{Q} / \mathbb{Z})=\operatorname{Hom}_{\mathrm{cts}}\left(G_{F}, \mathbb{Q} / \mathbb{Z}\right)$ be a character of $G_{F}$ with image $\mathbb{Z} / n \subseteq \mathbb{Q} / \mathbb{Z}$, represented by a pair $(L / F, \sigma)$; i.e., $L / F$ is a finite cyclic Galois extension of degree $n$ such that

$$
G_{L}=\operatorname{Ker}\left(\chi: G_{F} \rightarrow \mathbb{Q} / \mathbb{Z}\right)
$$

and $\sigma \in \operatorname{Gal}(L / F)$ is a generator such that $\chi(\sigma)=1+n \mathbb{Z} \in \mathbb{Z} / n$. Recall that [Gille and Szamuely 2006, §2.5] the cyclic algebra $(\chi, b)$ associated with $\chi$ and an element $b \in F^{*}$ is the $F$-algebra generated by $L$ and a word $y$ subject to the following multiplication relations:

$$
y^{n}=b \quad \text { and } \quad \lambda y=y \sigma(\lambda) \quad \text { for all } \lambda \in L .
$$

It is a standard fact that $(\chi, b)$ is a central simple algebra of degree $n$ over $F$. The class of the cyclic algebra $(\chi, b)$ in $\operatorname{Br}(F)[n]=H^{2}\left(F, \mu_{n}\right)$ coincides with the cup product of $\chi \in H^{1}(F, \mathbb{Z} / n)$ and $(b) \in H^{1}\left(F, \mu_{n}\right)$. 
If $\mu_{n} \subseteq F$, then by Kummer theory $L$ is of the form $L=F(\sqrt[n]{a})$ for some $a \in F^{*}$. There is a primitive $n$-th root of unity $\xi_{n} \in F$ such that $\sigma(\sqrt[n]{a})=\xi_{n} \sqrt[n]{a}$. The cyclic algebra $(\chi, b)$ is isomorphic to the $F$-algebra $(a, b)_{\xi_{n}}$, which by definition is the $F$-algebra generated by two words $x, y$ subject to the relations

$$
x^{n}=a, \quad y^{n}=b \quad \text { and } \quad x y=\xi_{n} y x .
$$

Conversely, when $F$ contains a primitive $n$-th root of unity $\xi_{n}$, the algebra $(a, b)_{\xi_{n}}$ associated to elements $a, b \in F^{*}$ is isomorphic to $(\chi, b)$, where $\chi \in H^{1}(F, \mathbb{Q} / \mathbb{Z})$ is the character represented by the cyclic extension $L / F=F(\sqrt[n]{a}) / F$ and the $F$-automorphism $\sigma \in \operatorname{Gal}(L / F)$ that sends $\sqrt[n]{a}$ to $\xi_{n} \sqrt[n]{a}$. The class of the algebra $(a, b)_{\xi_{n}}$ in $\operatorname{Br}(F)$ will be denoted by $(a, b)$ when the degree $n$ and the choice of $\xi_{n} \in F$ are clear from the context. This notation is compatible with the notion of symbol classes via the isomorphism $\operatorname{Br}(F)[n]=H^{2}\left(F, \mu_{n}\right) \cong H^{2}\left(F, \mu_{n}^{\otimes 2}\right)$ corresponding to the choice of $\xi_{n} \in F$.

\section{Division algebras over local henselian surfaces}

In this section, we first recall a number of techniques in Saltman's method of detecting ramification of division algebras [Saltman 1997; 2007] and then we will prove Theorem 1.3.

Ramification of division algebras over surfaces. In this subsection, let $X$ be a regular excellent surface and let $F$ be the function field of $X$. By resolution of embedded singularities [Shafarevich 1966, Theorem on p. 38 and Remark on p. 43; Lipman 1975, p. 193], for any effective divisor $D$ on $X$, there exists a regular surface $X^{\prime}$ together with a proper birational morphism $X^{\prime} \rightarrow X$, obtained by a sequence of blow-ups, such that the total transform $D^{\prime}$ of $D$ in $X^{\prime}$ is a simple normal crossing (snc) divisor (i.e., the reduced subschemes on the irreducible components of $D^{\prime}$ are regular curves and they meet transversally everywhere). We will use this result without further reference.

Let $n$ be a positive integer that is invertible on $X$, and let $\alpha \in \operatorname{Br}(F)[n]$ be a Brauer class of order dividing $n$. For any discrete valuation $v \in \Omega(F / X)$, let $\operatorname{ram}_{v}$ denote the ramification map (or the residue map)

$$
\operatorname{ram}_{v}=\partial_{v}^{2,1}: \operatorname{Br}(F)[n]=H^{2}\left(F, \mu_{n}\right) \rightarrow H^{1}(\kappa(v), \mathbb{Z} / n) .
$$

If $v=v_{C}$ is the discrete valuation centered at the generic point of a curve $C \subseteq X$, we write $\operatorname{ram}_{C}=\operatorname{ram}_{v_{C}}$. The ramification locus of $\alpha \in \operatorname{Br}(F)[n]$ on $X$, denoted $\operatorname{Ram}_{X}(\alpha)$, is by definition the (finite) union of curves $C \subseteq X$ such that $\operatorname{ram}_{C}(\alpha) \neq 0 \in H^{1}(\kappa(C), \mathbb{Z} / n)$. The ramification divisor of $\alpha$ on $X$, denoted again by $\operatorname{Ram}_{X}(\alpha)$ by abuse of notation, is the reduced divisor supported on the ramification locus. After several blow-ups, we may assume $\operatorname{Ram}_{X}(\alpha)$ is an snc divisor on $X$. 
Definition 3.1 [Saltman 2007, §2]. Let $X, F$ and $\alpha$ be as above. Assume that $\operatorname{Ram}_{X}(\alpha)$ is an snc divisor on $X$. A closed point $P \in X$ is called

(1) a distant point for $\alpha$ if $P \notin \operatorname{Ram}_{X}(\alpha)$,

(2) a curve point for $\alpha$ if $P$ lies on one and only one irreducible component of $\operatorname{Ram}_{X}(\alpha)$ and

(3) a nodal point for $\alpha$ if $P$ lies on two different irreducible components of $\operatorname{Ram}_{X}(\alpha)$.

Saltman essentially derived the following theorem from a local study of a Brauer class at closed points in its ramification locus [Saltman 1997, Proposition 1.2]:

Theorem 3.2 [Saltman 1997, Theorem 2.1]. Let X be a regular excellent surface that is quasiprojective over a ring, $F$ the function field of $X, n>0$ a positive integer that is invertible on $X$ and $\alpha \in \operatorname{Br}(F)[n]$. Assume $\mu_{n} \subseteq F$.

Then there exist $f, g \in F^{*}$ such that the field extension $M / F:=F(\sqrt[n]{f}, \sqrt[n]{g}) / F$ splits all ramification of $\alpha$ over $X$, i.e., $\alpha_{M} \in \mathrm{Br}_{\mathrm{nr}}(M / X)$.

(Although our setup here differs from that of Saltman's Theorem 2.1, a careful verification shows that his proof - with Gabber's corrections given in [Saltman 1998] - still works. One can also find a proof of Theorem 3.2 in [Brussel 2010, Lemma 7.8]. When $n$ is prime, a stronger statement holds; see [Saltman 2008, Theorem 7.13] and Proposition 3.11.)

Remark 3.3. Let $R$ be a two-dimensional, henselian, excellent local domain with fraction field $K$ and residue field $k$. By resolution of singularities for surfaces (see [Lipman 1975; 1978]), there exists a regular proper model $\mathscr{L} \rightarrow \operatorname{Spec} R$. The structural morphism $\mathscr{X} \rightarrow \operatorname{Spec} R$ is actually projective by [Grothendieck 1967, IV.21.9.13]. So Theorem 3.2 applies to such a regular proper model $\mathscr{X} \rightarrow \operatorname{Spec} R$.

If the residue field $k$ of $R$ is finite, Theorem 3.2 has the following refined form over the fraction field $K$ :

Let $n>0$ be a positive integer that is invertible in the finite residue field $k$. Assume that $\mu_{n} \subseteq R$. Then for any finite collection of Brauer classes $\alpha_{i} \in \operatorname{Br}(K)[n]$, $1 \leq i \leq m$, there exist $f, g \in K^{*}$ such that the field extension $M / K:=K(\sqrt[n]{f}, \sqrt[n]{g}) / K$ splits all the $\alpha_{i}, i=1, \ldots, m$.

In the literature, this result has been established in the case where $K$ is a function field of a $p$-adic curve and where $n$ is a prime number, and the proof is essentially an observation of Gabber and Colliot-Thélène [Colliot-Thélène 1998; Hoffmann and Van Geel 1998, Theorem 2.5]. One may verify that essentially the same arguments work in the local henselian case considered here.

We will need the following analog of [Saltman 1997, Theorem 3.4] in the sequel: 
Theorem 3.4. Let $R$ be a two-dimensional, henselian, excellent local domain with fraction field $K$ and residue field $k$. Let $n>0$ be a positive integer that is invertible in $k$. Assume that $k$ is a $B_{1}$ field.

Then any Brauer class $\alpha \in \operatorname{Br}(K)$ of order $n$ has index dividing $n^{2}$.

Proof. This follows on parallel lines along the proof of [Saltman 1997, Theorem 3.4] with suitable substitutions of the ingredients used in the case of $p$-adic function fields. For the sake of the reader's convenience, we recall the argument.

We may assume $n=q^{r}$ is a power of a prime number $q$. Let $\mathscr{X} \rightarrow \operatorname{Spec} R$ be a regular proper model. For any finite field extension $K^{\prime} / K$, the integral closure $R^{\prime}$ of $R$ in $K^{\prime}$ satisfies the same assumptions as $R$ and $K^{\prime}$ is the function field of a regular proper model $\mathscr{X}^{\prime}$ of Spec $R^{\prime}$. One has $\Omega\left(K^{\prime} / \mathscr{X}^{\prime}\right)=\Omega\left(K^{\prime} / \mathscr{X}\right)$ and $\operatorname{Br}_{\mathrm{nr}}\left(K^{\prime} / \mathscr{X}\right)=\operatorname{Br}_{\mathrm{nr}}\left(K^{\prime} / \mathscr{X}^{\prime}\right)=0$ by Theorem 2.1(iii) and Corollary 2.5. So it suffices to find a finite separable field extension $K^{\prime} / K$ of degree $q^{2 r} m$ with $q \nmid m$ such that $K^{\prime} / K$ splits all ramification of $\alpha$ over $\mathscr{X}$.

Now we proceed by induction on $r$. First assume $r=1$. Then the result is immediate from Theorem 3.2 if $\mu_{q} \subseteq F$. The general case follows by passing to the extension $F\left(\mu_{q}\right) / F$, which has degree prime to $q$.

For general $r$, the inductive hypothesis applied to the Brauer class $q \alpha$ implies that there is a separable field extension $K^{\prime} / K$ splitting all ramification of $q \alpha$ over $\mathscr{X}$, which has degree $q^{2 r-2} m^{\prime}$, where $q \nmid m^{\prime}$. But $q \alpha_{K^{\prime}}=0 \in \operatorname{Br}\left(K^{\prime}\right)$ by Corollary 2.5. By the case with $r=1$, we can find a separable extension $K^{\prime \prime} / K^{\prime}$ of degree $q^{2} m^{\prime \prime}$ with $q \nmid m^{\prime \prime}$ that splits all ramification of $\alpha_{K^{\prime}}$ over $\mathscr{X}$. Now $K^{\prime \prime} / K$ is a separable extension of degree $\left[K^{\prime \prime}: K\right]=q^{2 r} m$ with $m=m^{\prime} m^{\prime \prime}$ coprime to $q$ and $K^{\prime \prime} / K$ splits all ramification of $\alpha$ over $\mathscr{X}$, as desired.

We will give an example of a Brauer class $\alpha \in \operatorname{Br}(K)$ of order $n$ that is of index $n^{2}$ in Example 3.18.

Classification of nodal points. To prove further results, we need more analysis on ramification at nodal points, for which we briefly recall in this subsection some basic notions and results due to Saltman. The reader is referred to [Saltman 2007, $\S \S 2-3]$ or [Brussel 2010, §§7-8] for more details.

Let $X$ be a regular excellent surface with function field $F$, and let $q$ be a prime number that is invertible on $X$. Let $\alpha \in \operatorname{Br}(F)[q]$. Assume that $\operatorname{Ram}_{X}(\alpha)$ is an snc divisor on $X$. Let $P \in X$ be a nodal point for $\alpha$ (Definition 3.1), lying on two distinct irreducible components $C_{1}$ and $C_{2}$ of $\operatorname{Ram}_{X}(\alpha)$. Let $\chi_{1}=\operatorname{ram}_{C_{1}}(\alpha)$ and $\chi_{2}=\operatorname{ram}_{C_{2}}(\alpha)$ be respectively the ramifications of $\alpha$ at $C_{1}$ and $C_{2}$. Since the natural sequence induced by residue maps

$$
H^{2}\left(F, \mu_{q}\right) \rightarrow \bigoplus_{v \in\left(\operatorname{Spec} \mathcal{O}_{X, P}\right)^{(1)}} H^{1}(\kappa(v), \mathbb{Z} / q) \rightarrow H^{0}\left(\kappa(P), \mu_{q}^{\otimes(-1)}\right)
$$


is a complex (see [Kato 1986] or [Colliot-Thélène 2006, Proposition 2.3]), $\chi_{1}=$ $\operatorname{ram}_{C_{1}}(\alpha) \in H^{1}\left(\kappa\left(C_{1}\right), \mathbb{Z} / q\right)$ is unramified at $P$ if and only if $\chi_{2}=\operatorname{ram}_{C_{2}} \in$ $H^{1}\left(\kappa\left(C_{2}\right), \mathbb{Z} / q\right)$ is unramified at $P$.

Definition 3.5 [Saltman 2007, §§2-3]. Let $X, F, q, \alpha$ and so on be as above. Assume that $\operatorname{Ram}_{X}(\alpha)$ is an snc divisor on $X$. Let $P \in X$ be a nodal point for $\alpha$, lying on two distinct irreducible components $C_{1}$ and $C_{2}$ of $\operatorname{Ram}_{X}(\alpha)$.

(1) $P$ is called a cold point for $\alpha$ if $\chi_{1}=\operatorname{ram}_{C_{1}}(\alpha) \in H^{1}\left(\kappa\left(C_{1}\right), \mathbb{Z} / q\right)$ (and hence also $\left.\chi_{2}=\operatorname{ram}_{C_{2}}(\alpha) \in H^{1}\left(\kappa\left(C_{2}\right), \mathbb{Z} / q\right)\right)$ is ramified at $P$.

(2) Assume now $\chi_{1}$ and $\chi_{2}$ are unramified at $P$ so that they lie respectively in $H^{1}\left(\mathscr{O}_{C_{1}, P}, \mathbb{Z} / q\right)$ and $H^{1}\left(\mathcal{O}_{C_{2}, P}, \mathbb{Z} / q\right)$. Let $\chi_{i}(P) \in H^{1}(\kappa(P), \mathbb{Z} / q), i=1,2$, be their specializations and $\left\langle\chi_{i}(P)\right\rangle, i=1,2$, be the subgroups of $H^{1}(\kappa(P), \mathbb{Z} / q)$ generated by $\chi_{i}(P)$, respectively. Then $P$ is called

(a) a cool point for $\alpha$ if $\left\langle\chi_{1}(P)\right\rangle=\left\langle\chi_{2}(P)\right\rangle=0$,

(b) a chilly point for $\alpha$ if $\left\langle\chi_{1}(P)\right\rangle=\left\langle\chi_{2}(P)\right\rangle \neq 0$ and

(c) a hot point for $\alpha$ if $\left\langle\chi_{1}(P)\right\rangle \neq\left\langle\chi_{2}(P)\right\rangle$.

When $P$ is a chilly point, there is a unique $s=s\left(C_{2} / C_{1}\right) \in(\mathbb{Z} / q)^{*}$ such that

$$
\chi_{2}(P)=s \cdot \chi_{1}(P) \in H^{1}(\kappa(P), \mathbb{Z} / q) .
$$

One says that $s=s\left(C_{2} / C_{1}\right)$ is the coefficient of the chilly point $P$ with respect to $C_{1}$.

Remark 3.6. One may verify without much pain that our classification of nodal points, following [Brussel 2010, Definition 8.5], is equivalent to Saltman's original formulation, which goes as follows. First consider the case $\mu_{q} \subseteq F$. Then

$$
\alpha \equiv(u, \pi)+(v, \delta)+r .(\pi, \delta)\left(\bmod \operatorname{Br}\left(\mathscr{O}_{X, P}\right)\right)
$$

by [Saltman 1997, Proposition 1.2]. Here $u, v \in \mathbb{O}_{X, P}^{*}, r \in \mathbb{Z} / q$ and $\pi, \delta \in \mathcal{O}_{X, P}$ are local equations of the two components of $\operatorname{Ram}_{X}(\alpha)$ passing through $P$. The point $P$ is a cold point if $r \neq 0 \in \mathbb{Z} / q$. Assume next $r=0 \in \mathbb{Z} / q$. Then $P$ is a cool point if $u(P), v(P) \in \kappa(P)^{* q}$, a chilly point if $u(P), v(P) \notin \kappa(P)^{* q}$ and they generate the same subgroup of $\kappa(P)^{*} / \kappa(P)^{* q}$ or a hot point otherwise. In the general case, let $X^{\prime} \rightarrow X$ be the connected finite étale cover obtained by adjoining all $q$-th roots of unity and let $\alpha^{\prime}$ be the canonical image of $\alpha$ in $\operatorname{Br}\left(F^{\prime}\right)$, where $F^{\prime}$ denotes the function field of $X^{\prime}$. Then for any two points $P_{1}^{\prime}, P_{2}^{\prime} \in X^{\prime}$, both lying over $P \in X, P_{1}^{\prime}$ is a cold, cool, chilly or hot point for $\alpha^{\prime}$ if and only if $P_{2}^{\prime}$ is, and in that case, one says that $P$ is a cold, cool, chilly or hot point for $\alpha$, respectively. When $P$ is chilly, the coefficient of $P$ with respect to a component through it is also well-defined, as the coefficient of any preimage $P^{\prime}$ of $P$.

To get some compatibility for coefficients of chilly points, one has to eliminate the so-called chilly loops, i.e., loops in the following graph. The set of vertices 
is the set of irreducible components of $\operatorname{Ram}_{X}(\alpha)$, and the number $r \geq 0$ of edges linking two vertices is equal to the number of chilly points in the intersection of the two curves corresponding to the two vertices. (Two vertices may be joined by two or more edges and thus contribute to some loops.)

Proposition 3.7 [Saltman 2007, Proposition 3.8]. Let $X, F, q$ and $\alpha \in \operatorname{Br}(F)[q]$ be as above. Assume that $\operatorname{Ram}_{X}(\alpha)$ is an snc divisor on $X$. Then there exists a proper birational morphism $X^{\prime} \rightarrow X$, obtained by a finite number of blow-ups, such that $\alpha$ has no cool points and no chilly loops on $X^{\prime}$.

We also need the notion of residual class at a ramified place. Let $C$ be an irreducible component of $\operatorname{Ram}_{X}(\alpha)$, and let $v=v_{C}$ be the associated discrete valuation of $F$. Choose any $x \in F^{*}$ with $q \nmid v(x)$ so that the extension $M / F:=F(\sqrt[q]{x}) / F$ is totally ramified at $v=v_{C}$ and $\alpha_{M}=\alpha \otimes_{F} M \in \operatorname{Br}(M)$ is unramified at the unique discrete valuation $w$ of $M$ that lies over $v$. One has $\kappa(w)=\kappa(v)=\kappa(C)$ and hence a well-defined Brauer class $\beta_{C, x} \in \operatorname{Br}(\kappa(C))$ given by the specialization of $\alpha_{M} \in \operatorname{Br}(M)$ in $\operatorname{Br}(\kappa(w))=\operatorname{Br}(\kappa(C))$. Let $(L / \kappa(C), \sigma)=\operatorname{ram}_{C}(\alpha)$ be the ramification of $\alpha$ at $C$. Whether $\beta_{C, x} \in \operatorname{Br}(\kappa(C))$ is split by the field extension $L / \kappa(C)$ does not depend on the choice of $M=F(\sqrt[q]{x})$ [Saltman 2007, Corollary 0.7]. We say that the residual classes of $\alpha$ at $C$ are split by the ramification if, for one (and hence for all) choice of $M=F(\sqrt[q]{x})$, the residual class $\beta_{C, x} \in \operatorname{Br}(\kappa(C))$ is split by $L / \kappa(C)$ [Saltman 2007, p. 821 Remark]. When we are only interested in this property, we will simply write $\beta_{C}$ for $\beta_{C, x} \in \operatorname{Br}(\kappa(C))$ with respect to any choice of $x$.

It is proved in [Saltman 2007, Propositions 0.5 and 3.10(d)] that if $\alpha$ has index $q$, then all the residual classes $\beta_{C}$ of $\alpha$ at all components $C$ of $\operatorname{Ram}_{X}(\alpha)$ are split by the ramification and there are no hot points for $\alpha$ on $X$.

Splitting over a Kummer extension. Let $X$ be a reduced scheme that is projective over a ring. Let $\mathscr{P} \subseteq X$ be a finite set of closed points of $X$. Denote by $\mathscr{K}_{X}$ the sheaf of meromorphic functions on $X$, and set $\mathscr{P}^{*}:=\bigoplus_{P \in \mathscr{P}} \kappa(P)^{*}$. Let $\mathcal{O}_{X, \mathscr{P}}^{*}$ denote the kernel of the natural surjection of sheaves $0_{X}^{*} \rightarrow \mathscr{P}^{*}$ so that there is a natural exact sequence

$$
1 \rightarrow \mathrm{O}_{X, \mathscr{P}}^{*} \rightarrow \mathrm{O}_{X}^{*} \rightarrow \mathscr{P}^{*} \rightarrow 1 .
$$

Define subgroups $K_{\mathscr{P}}^{*} \subseteq H^{0}\left(X, \mathscr{K}_{X}^{*}\right)$ and $H_{\mathscr{P}}^{0}\left(X, \mathscr{K}_{X}^{*} / \mathcal{O}_{X}^{*}\right) \subseteq H^{0}\left(X, \mathscr{K}_{X}^{*} / \mathcal{O}_{X}^{*}\right)$ by

$$
\begin{aligned}
K_{\mathscr{P}}^{*} & :=\left\{f \in H^{0}\left(X, \mathscr{K}_{X}^{*}\right) \mid f \in \mathcal{O}_{X, P}^{*} \quad \text { for all } P \in \mathscr{P}\right\}, \\
H_{\mathscr{P}}^{0}\left(X, \mathscr{K}_{X}^{*} / \mathcal{O}_{X}^{*}\right) & :=\left\{D \in H^{0}\left(X, \mathscr{K}_{X}^{*} / \mathcal{O}_{X}^{*}\right) \mid \operatorname{Supp}(D) \cap \mathscr{P}=\varnothing\right\} .
\end{aligned}
$$

Consider the natural map

$$
\phi: K_{\mathscr{P}}^{*} \rightarrow H_{\mathscr{P}}^{0}\left(X, \mathscr{K}_{X}^{*} / \mathcal{O}_{X}^{*}\right) \oplus\left(\bigoplus_{P \in \mathscr{P}} \kappa(P)^{*}\right), \quad f \mapsto\left(\operatorname{div}_{X}(f), \bigoplus f(P)\right)
$$


Proposition 3.8 [Saltman 2007, Proposition 1.6]. With notation as above, there is a natural isomorphism

$$
H^{1}\left(X, \mathcal{O}_{X, \mathscr{P}}^{*}\right) \cong \frac{H_{\mathscr{P}}^{0}\left(X, \mathcal{K}_{X}^{*} / \mathcal{O}_{X}^{*}\right) \oplus\left(\bigoplus_{P \in \mathscr{P}} \kappa(P)^{*}\right)}{\phi\left(K_{\mathscr{P}}^{*}\right)} .
$$

The analog in the arithmetic case of the following proposition is [Saltman 2007, Proposition 1.7]. The following generalization to the case where $A$ is twodimensional will be indispensable in the proofs of our results:

Proposition 3.9. Let $A$ be a (noetherian) normal, henselian local domain with residue field $\kappa, X$ an integral scheme and $X \rightarrow$ Spec $A$ a proper morphism whose closed fiber $X_{0}$ has dimension $\leq 1$ and whose generic fiber is geometrically integral. Let $m$ be a positive integer invertible in A. Let $\bar{X}=\left(X_{0}\right)_{\text {red }}$ be the reduced closed subscheme on the closed fiber $X_{0}$. Suppose that $\bar{X}$ is geometrically reduced over $\kappa$ (e.g., $\kappa$ is perfect). Then for any finite set $\mathscr{P}$ of closed points of $X$, the natural map

$$
H^{1}\left(X, O_{X, \mathscr{P}}^{*}\right) \rightarrow H^{1}\left(\bar{X}, \mathrm{O}_{\bar{X}, \mathscr{P}}^{*}\right)
$$

is surjective and induces a canonical isomorphism

$$
H^{1}\left(X, O_{X, \mathscr{P}}^{*}\right) / m \cong H^{1}\left(\bar{X}, O_{\bar{X}, \mathscr{P}}^{*}\right) / m
$$

To prove the proposition, we need a well-known lemma.

Lemma 3.10. Let $A$ be a (noetherian) henselian local ring, $X \rightarrow$ Spec $A$ a proper morphism with closed fiber $X_{0}$ of dimension $\leq 1, m>0$ a positive integer that is invertible in $A$ and $\bar{X}=\left(X_{0}\right)_{\text {red }}$ the reduced closed subscheme on the closed fiber $X_{0}$.

Then the natural map $\operatorname{Pic}(X) \rightarrow \operatorname{Pic}(\bar{X})$ is surjective and induces an isomorphism

$$
\operatorname{Pic}(X) / m \stackrel{\sim}{\rightarrow} \operatorname{Pic}(\bar{X}) / m \text {. }
$$

Proof. The surjectivity of $\operatorname{Pic}(X) \rightarrow \operatorname{Pic}(\bar{X})$ follows from [Grothendieck 1967, IV.21.9.12]. Then the commutative diagram with exact rows, which comes from the Kummer sequence,

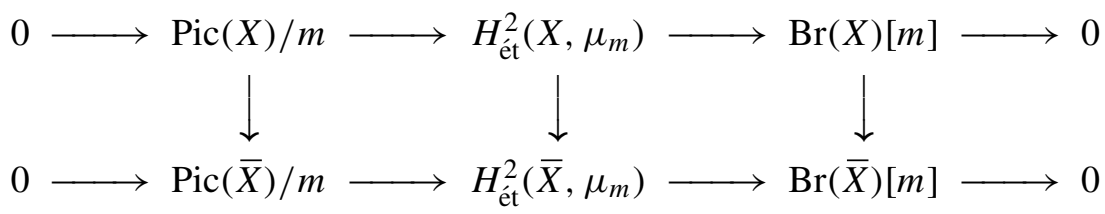

yields the desired isomorphism $\operatorname{Pic}(X) / m \stackrel{\sim}{\rightarrow} \operatorname{Pic}(\bar{X}) / m$ since the vertical map in the middle is an isomorphism by proper base change [Milne 1980, p. 224, Corollary 2.7], noticing also that any scheme $Y$ has the same étale cohomology with value in a commutative étale group scheme as its reduced closed subscheme $Y_{\text {red }}$ [SGA 4.2 1972, Exposé VIII, Corollary 1.2]. 
Proof of Proposition 3.9. Consider the commutative diagram with exact rows

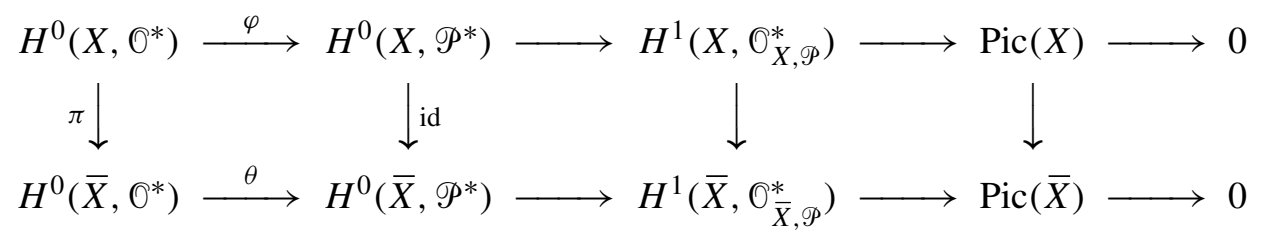

from which the surjectivity of $H^{1}\left(X, \mathcal{O}_{X, \mathscr{P}}^{*}\right) \rightarrow H^{1}\left(\bar{X}, \mathcal{O}_{\bar{X}, \mathscr{P}}^{*}\right)$ is immediate since $\operatorname{Pic}(X) \rightarrow \operatorname{Pic}(\bar{X})$ is surjective by Lemma 3.10. Put $M:=\Im(\varphi) \subseteq N:=\Im(\theta)$.

We claim that $\pi$ is surjective. Indeed, by Zariski's connectedness theorem [Grothendieck 1961, III.4.3.12], the hypotheses that $A$ is normal and the generic fiber of $X \rightarrow \operatorname{Spec} A$ is geometrically integral imply that the closed fiber $X_{0}$ is geometrically connected. The reduced closed fiber $\bar{X}=\left(X_{0}\right)_{\text {red }}$ is geometrically connected as well. Since $\bar{X}$ is assumed to be geometrically reduced, we have $H^{0}\left(\bar{X}, \mathrm{O}^{*}\right)=\kappa^{*}$. Thus, the map $\pi: H^{0}\left(X, \mathcal{O}^{*}\right) \rightarrow H^{0}\left(\bar{X}, \mathrm{O}^{*}\right)$ is clearly surjective since $A^{*} \subseteq H^{0}\left(X, O^{*}\right)$.

Now our claim shows that $M=N$, and then it follows that

$$
\operatorname{Ker}\left(H^{1}\left(X, \mathcal{O}_{X, \mathscr{P}}^{*}\right) \rightarrow H^{1}\left(\bar{X}, \mathcal{O}_{\bar{X}, \mathscr{P}}^{*}\right)\right) \cong B:=\operatorname{Ker}(\operatorname{Pic}(X) \rightarrow \operatorname{Pic}(\bar{X})) .
$$

It's sufficient to show $B / m=0$. From the commutative diagram with exact rows

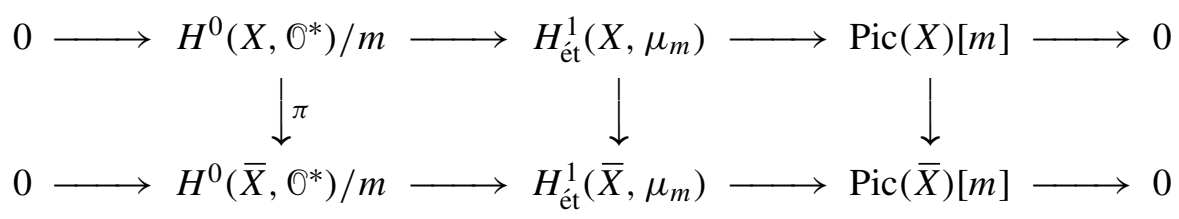

it follows that $\operatorname{Pic}(X)[m] \cong \operatorname{Pic}(\bar{X})[m]$ since the vertical map in the middle is an isomorphism by proper base change and the left vertical map is already shown to be surjective. Now applying the snake lemma to the commutative diagram

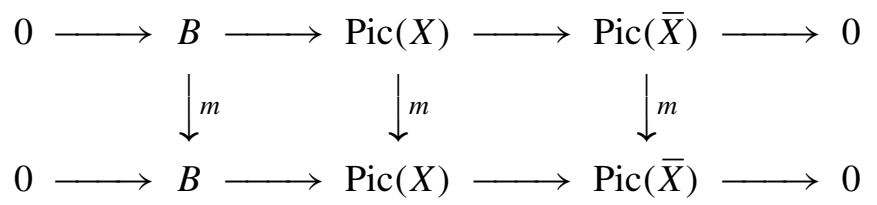

and using Lemma 3.10, we easily find $B / m=0$, which completes the proof.

The following result is proved in [Saltman 2008, Theorem 7.13] in the case where $\mu_{q} \subseteq F$ without assuming the residue field $\kappa$ perfect. It says essentially that the conclusion of Theorem 3.2 can be strengthened for Brauer classes of prime order.

Proposition 3.11. Let $A$ be a (noetherian) henselian local domain with residue field $\kappa, q$ a prime number unequal to the characteristic of $\kappa$ and $X$ a regular excellent surface equipped with a proper dominant morphism $X \rightarrow \operatorname{Spec} A$ whose 
closed fiber is of dimension $\leq 1$. Let $F$ be the function field of $X$ and $\alpha \in \operatorname{Br}(F)[q]$. Assume that $\kappa$ is perfect and that $\alpha$ has index $q$.

Then there is some $g \in F^{*}$ such that the field extension $M / F:=F(\sqrt[q]{g}) / F$ splits all ramification of $\alpha$ over $X$, i.e., $\alpha_{M} \in \mathrm{Br}_{\mathrm{nr}}(M / X)$.

Proof. Replacing $A$ by its normalization if necessary, we may assume that $A$ is normal. Let $\operatorname{Ram}_{X}(\alpha)=\sum C_{i}$ be the ramification divisor of $\alpha$ on $X$, and let $\bar{X}=\left(X_{0}\right)_{\text {red }}$ be the reduced closed subscheme on the closed fiber $X_{0}$. After a finite number of blow-ups, we may assume that $X_{0}$ is purely of dimension 1 , that $B:=\left(\operatorname{Ram}_{X}(\alpha) \cup \bar{X}\right)_{\text {red }}$ is an snc divisor and that there are no cool points or chilly loops for $\alpha$ on $X$ (Proposition 3.7). Write

$$
\left(L_{i} / \kappa\left(C_{i}\right), \sigma_{i}\right)=\operatorname{ram}_{C_{i}}(\alpha) \in H^{1}\left(\kappa\left(C_{i}\right), \mathbb{Z} / q\right)
$$

for the ramification of $\alpha$ at $C_{i}$. By the assumption on the index, there are no hot points for $\alpha$ on $X$ and the residual classes of $\alpha$ at $C_{i}$ are split by the ramification $L_{i} / \kappa\left(C_{i}\right)$ for every $i$ [Saltman 2007, Propositions 0.5 and 3.10(d)]. Using [Saltman 2007, Theorem 4.6], we can find $\pi \in F^{*}$ having the following properties:

(P1) The valuation $v_{C_{i}}(\pi)=s_{i}$ is not divisible by $q$.

(P2) If $P$ is a chilly point in the intersection of $C_{i}$ and $C_{j}$, then the coefficient $s\left(C_{j} / C_{i}\right)$ of $P$ with respect to $C_{i}$ (Definition 3.5) satisfies $s\left(C_{j} / C_{i}\right) s_{i}=$ $s_{j} \in \mathbb{Z} / q \mathbb{Z}$.

(P3) The divisor $E:=\operatorname{div}_{X}(\pi)-\sum s_{i} C_{i}$ does not contain any singular points of $B=\left(\operatorname{Ram}_{X}(\alpha) \cup X_{0}\right)_{\text {red }}$ or any irreducible component of $B$ in its support.

(P4) With respect to $F^{\prime}:=F\left(\pi^{1 / q}\right)$, the residue Brauer classes $\beta_{C_{i}, F^{\prime}}=\beta_{C_{i}, \pi} \in$ $\operatorname{Br}\left(\kappa\left(C_{i}\right)\right)$ of $\alpha$ at all the $C_{i}$ are trivial.

(P5) For any closed point $P$ in the intersection of $E$ and some $C_{i}$, the intersection multiplicity $\left(C_{i} \cdot E\right)_{P}$ is a multiple of $q$ if the corresponding field extension $L_{i} / \kappa\left(C_{i}\right)$ is nonsplit at $P$.

Let $\gamma \in \operatorname{Pic}(X)$ be the class of $\mathrm{O}_{X}(-E)$, and let $\bar{\gamma} \in \operatorname{Pic}(\bar{X})$ be its canonical image. By property (P3), $E$ and $\bar{X}$ only intersect in nonsingular points of $\bar{X}$. So we can represent $\bar{\gamma}$ as a Cartier divisor on $\bar{X}$ using the intersection of $-E$ and $\bar{X}$. This divisor can be chosen in the form

$$
\sum q n_{j} Q_{j}+\sum n_{l} Q_{l}^{\prime},
$$

where $Q_{j}$ and $Q_{l}^{\prime}$ are nonsingular points on $\bar{X}$, and for each $Q_{l}^{\prime}$, one has either $Q_{l}^{\prime} \notin \operatorname{Ram}_{X}(\alpha)$ or $Q_{l}^{\prime} \in C_{i}$ for exactly one $C_{i}$ and the corresponding field extension $L_{i} / \kappa\left(C_{i}\right)$ is split at $Q_{l}^{\prime}$ (by property (P5)).

By [Grothendieck 1967, IV.21.9.11 and IV.21.9.12], there exists a prime divisor $E_{l}^{\prime}$ on $X$ such that $\left.E_{l}^{\prime}\right|_{\bar{X}}=Q_{l}^{\prime}$ as Cartier divisors on $\bar{X}$. Note that $E_{l}^{\prime} \nsubseteq \operatorname{Ram}_{X}(\alpha)$ 
because otherwise $Q_{l}^{\prime} \in E_{l}^{\prime} \cap \bar{X}$ would be a singular point of $E_{l}^{\prime} \cup \bar{X} \subseteq B=$ $\left(\operatorname{Ram}_{X}(\alpha) \cup \bar{X}\right)_{\text {red. }}$ Set $E^{\prime}=-E-\sum n_{l} E_{l}^{\prime}$. Let $\mathscr{P}$ be the set of all singular points of $B$ (in particular, $\mathscr{P}$ contains all nodal points for $\alpha$ ).

Let $\gamma^{\prime} \in H^{1}\left(X, O_{X, \mathscr{P}}^{*}\right)$ be the element represented by the pair

$$
\left(E^{\prime}, \bigoplus 1\right) \in H_{\mathscr{P}}^{0}\left(X, \mathscr{K}^{*} / O^{*}\right) \oplus\left(\bigoplus_{P \in \mathscr{P}} \kappa(P)^{*}\right)
$$

via the isomorphism

$$
H^{1}\left(X, O_{X, \mathscr{P}}^{*}\right) \cong \frac{H_{\mathscr{P}}^{0}\left(X, \mathscr{K}^{*} / \mathcal{O}^{*}\right) \oplus\left(\bigoplus_{P \in \mathscr{P}} \kappa(P)^{*}\right)}{K_{\mathscr{P}}^{*}}
$$

in Proposition 3.8. (Here $E^{\prime} \in H_{\mathscr{\rho}}^{0}\left(X, \mathscr{K}^{*} / \mathcal{O}^{*}\right)$ since by the choice of $\pi, E$ does not contain any singular points of $B=\left(\operatorname{Ram}_{X}(\alpha) \cup \bar{X}\right)_{\text {red. }}$. $)$ The image $\bar{\gamma}^{\prime}$ of $\gamma^{\prime}$ in $H^{1}\left(\bar{X}, \mathscr{O}_{\bar{X}, \mathscr{P}}^{*}\right)$ lies in $q \cdot H^{1}\left(\bar{X}, \mathcal{O}_{\bar{X}, \mathscr{P}}^{*}\right)$ by the expression (3-1).

From Proposition 3.9, it follows that $\gamma^{\prime} \in q \cdot H^{1}\left(X, O_{X, \mathscr{P}}^{*}\right)$. Thus, by Proposition 3.8, there is a divisor $E^{\prime \prime} \in H_{\mathscr{P}}^{0}\left(X, \mathscr{K}^{*} / \mathcal{O}^{*}\right)$, elements $a(P) \in \kappa(P)^{*}$ for each $P \in \mathscr{P}$ and $f \in F^{*}$ such that $f$ is a unit at every $P \in \mathscr{P}, \operatorname{div}_{X}(f)=E^{\prime}+q E^{\prime \prime}$ and $f(P)=a(P)^{q}$ for all $P \in \mathscr{P}$. We now compute

$$
\begin{aligned}
\operatorname{div}_{X}(f \pi) & =\operatorname{div}_{X}(f)+\operatorname{div}_{X}(\pi)=\left(E^{\prime}+q E^{\prime \prime}\right)+\left(\sum s_{i} C_{i}+E\right) \\
& =-E-\sum n_{l} E_{l}^{\prime}+q E^{\prime \prime}+E+\sum s_{i} C_{i} \\
& =\sum s_{i} C_{i}+\left(q E^{\prime \prime}-\sum n_{l} E_{l}^{\prime}\right) \\
& =: \sum s_{i} C_{i}+\sum \tilde{n}_{j} D_{j} .
\end{aligned}
$$

For any $D_{j}$, the following properties hold:

(P6) $D_{j}$ can only intersect $B$ in nonsingular points of $B$.

(P7) If $q \nmid \tilde{n}_{j}$, then $D_{j} \in\left\{E_{l}^{\prime}\right\}$ so that either $D_{j} \cap \operatorname{Ram}_{X}(\alpha)=\varnothing$ or $D_{j} \cap \operatorname{Ram}_{X}(\alpha)$ consists of a single point $P$ that lies on one $C_{i}$ and the corresponding field extension $L_{i} / \kappa\left(C_{i}\right)$ splits at $P$.

Now we claim that $g=f \pi$ satisfies the required property. That is, putting $M=F\left((f \pi)^{1 / q}\right), \alpha_{M} \in \operatorname{Br}(M)$ is unramified at every discrete valuation of $M$ that lies over a point or a curve on $X$.

Consider a discrete valuation of $M$ lying over some $v \in \Omega(F / X)$.

If $v$ is centered at some $C_{i}$, then $M / F$ is totally ramified at $v$ since the coefficient $s_{i}$ of $f \pi$ at $C_{i}$ is prime to $q$ (3-2); hence, in particular, $M / F$ splits the ramification of $\alpha$ at $v$. As $\alpha$ is unramified at all other curves on $X$, we may restrict to the case where $v$ is centered at a closed point $P$ of $X$. By [Saltman 2007, Theorem 3.4], we can also ignore distant points and curve points $P \in C_{i}$ where $L_{i} / \kappa\left(C_{i}\right)$ splits at $P$. 
Now assume that $P$ is a curve point lying on some $C_{1} \in\left\{C_{i}\right\}$ where the corresponding field extension $L_{1} / \kappa\left(C_{1}\right)$ is nonsplit at $P$. By property (P7), the only curves other than $C_{1}$ in the support of $\operatorname{div}_{X}(f \pi)$ that can pass through $P$ have coefficients a multiple of $q$. Therefore, in $R_{P}=O_{X, P}$, we have $f \pi=u \pi_{1}^{s_{1}} \delta^{q}$ with $u \in R_{P}^{*}, \pi_{1} \in R_{P}$ a uniformizer of $C_{1}$ at $P$ and $\delta \in R_{P}$ prime to $\pi_{1}$. Using [Saltman 2007, Proposition 3.5], we then conclude that $M / F$ splits all ramification of $\alpha$ at $v$.

Recall that we have assumed there are no cool points or hot points for $\alpha$. So in the only remaining cases, $P$ is either a cold point or a chilly point.

Assume first that $P$ is a cold point for $\alpha$. By property (P4) and [Saltman 2007, Corollary 0.7], the residual class $\beta_{C_{i}, M}$ of $\alpha$ at any $C_{i}$ with respect to $M=F\left((f \pi)^{1 / q}\right)$ is given by the class of a cyclic algebra $\left(\chi_{i}, \bar{f}^{-t}\right)$, where

$$
\chi_{i}=\left(L_{i} / \kappa\left(C_{i}\right), \sigma_{i}\right)=\operatorname{ram}_{C_{i}}(\alpha) \in H^{1}\left(\kappa\left(C_{i}\right), \mathbb{Z} / q\right),
$$

$t$ is an integer prime to $q$ and $\bar{f}$ denotes the canonical image of $f$ in $\kappa\left(C_{i}\right)$. Since $f$ is a $q$-th power in $\kappa(P)$ by the choice, it follows easily that $\beta_{C_{i}, M}$ is unramified at $P$. In the local ring $R_{P}=\mathcal{O}_{X, P}$, we have $f \pi=u_{P} \pi_{1}^{s_{1}} \pi_{2}^{s_{2}}$ for some $u_{P} \in R_{P}^{*}$ by (3-2) and property (P6). Hence, by [Saltman 2007, Proposition 3.10(c)], $M$ splits all ramification of $\alpha$ at $v$.

Finally, consider the case where $P$ is a chilly point. Let $C_{1}, C_{2} \in\left\{C_{i}\right\}$ be the two different irreducible components of $\operatorname{Ram}_{X}(\alpha)$ through $P$, and let $\pi_{1}, \pi_{2} \in R_{P}=\mathbb{O}_{X, P}$ be uniformizers of $C_{1}$ and $C_{2}$ at $P$. Again by (3-2) and property (P6), we have $f \pi=u_{P} \pi_{1}^{s_{1}} \pi_{2}^{s_{2}}$ for some $u_{P} \in R_{P}^{*}$. Let $s=s\left(C_{2} / C_{1}\right)$ be the coefficient of $P$ with respect to $C_{1}$. Using property ( $\left.\mathrm{P} 2\right)$, we find that $M=F\left((f \pi)^{1 / q}\right)$ may be written in the form $M=F\left(\left(\pi_{1}^{\prime} \pi_{2}^{s}\right)^{1 / q}\right)$, where $\pi_{1}^{\prime} \in R_{P}$ is a uniformizer of $C_{1}$ at $P$. Thus, by [Saltman 2007, Proposition 3.9(a)], $M / F$ splits all ramification of $\alpha$ at $v$, which completes the proof.

Corollary 3.12. Let $A$ be a (noetherian) henselian local domain with residue field $\kappa, q$ a prime number unequal to the characteristic of $\kappa$, and $X$ a regular excellent surface equipped with a proper dominant morphism $X \rightarrow \operatorname{Spec} A$ whose closed fiber is of dimension $\leq 1$. Let $F$ be the function field of $X$ and $\alpha \in \operatorname{Br}(F)[q]$. Assume that $\kappa$ is a $B_{1}$ field and that $\alpha$ has index $q$.

If either $\mu_{q} \subseteq F$ or $\kappa$ is perfect, then $\alpha$ is represented by a cyclic algebra of degree $q$.

Proof. If $\mu_{q} \subseteq F$, we may use [Saltman 2008, Theorem 7.13] to find a degree- $q$ Kummer extension $M / F=F(\sqrt[q]{g}) / F$ that splits all ramification of $\alpha$ over $X$. If $\kappa$ is perfect, such an extension exists by Proposition 3.11. As in the proof of Theorem 3.4, we have $\mathrm{Br}_{\mathrm{nr}}(M / X)=0$ by Corollary 2.5. Hence, $\alpha_{M}=0 \in \operatorname{Br}(M)$. Then by a theorem of Albert [Saltman 2007, Proposition 0.1], which is rather 
immediate when assuming the existence of a primitive $q$-th root of unity, $\alpha$ is represented by a cyclic algebra of degree $q$.

Recall that $R$ always denotes a two-dimensional, henselian, excellent local domain with fraction field $K$ and residue field $k$. Applying Corollary 3.12 to a regular proper model $\mathscr{L} \rightarrow \operatorname{Spec} R$ yields the following:

Theorem 3.13. Assume that the residue field $k$ of $R$ has property $B_{1}$. Let $q$ be a prime number unequal to the characteristic of $k$.

If either $\mu_{q} \subseteq R$ or $k$ is perfect, then any Brauer class $\alpha \in \operatorname{Br}(K)[q]$ of index $q$ is represented by a cyclic algebra of degree $q$.

Remark 3.14. (1) In Proposition 3.11 or Corollary 3.12, according to the above proof, if we assume the morphism $X \rightarrow \operatorname{Spec} A$ is chosen such that $\operatorname{Ram}_{X}(\alpha)$ is an snc divisor and that $\alpha$ has no cool points or chilly loops on $X$, then the hypothesis that $\alpha$ has index $q$ may be replaced by the weaker condition that all the residual classes $\beta_{C}$ of $\alpha$ at all components $C$ of $\operatorname{Ram}_{X}(\alpha)$ are split by the ramification.

(2) Similarly, let $\mathscr{X} \rightarrow \operatorname{Spec} R$ be a regular proper model such that $\operatorname{Ram}_{\mathscr{X}}(\alpha)$ is an snc divisor and that $\alpha \in \operatorname{Br}(K)[q]$ has no cool points or chilly loops on $\mathscr{X}$. Then the conclusion in Theorem 3.13 remains valid if instead of assuming $\alpha$ has index $q$ we only require that all the residual classes of $\alpha$ at all components of $\operatorname{Ram}_{\mathscr{L}}(\alpha)$ are split by the ramification.

(3) In the context of Theorem 3.13, if $k$ is a separably closed field, [Colliot-Thélène et al. 2002, Theorem 2.1] proved a stronger result: any Brauer class $\alpha \in \operatorname{Br}(K)$ of order $n$ that is invertible in $R$ (but not necessarily a prime) is represented by a cyclic algebra of index $n$.

Some corollaries. As applications of results obtained previously, we give a criterion for $\alpha \in \operatorname{Br}(K)[q]$ to have index $q$. Also, we will prove Theorem 1.3.

We begin with the following easy and standard fact:

Lemma 3.15. Let $R$ be a two-dimensional, henselian, excellent local domain with fraction field $K$ and residue field $k$. Let $\mathscr{X} \rightarrow \operatorname{Spec} R$ be a regular proper model. Then for any curve $C \subseteq \mathscr{X}$, one has either

(i) $C$ is a proper curve over $k$ or

(ii) $C=\operatorname{Spec} B$, where $B$ is a domain whose normalization $B^{\prime}$ is a henselian discrete valuation ring with residue field finite over $k$.

Proof. After replacing $R$ by its normalization, we may assume $R$ is normal.

Consider the scheme-theoretic image $D$ of $C \subseteq \mathscr{X}$ by the structural morphism $\mathscr{L} \rightarrow \operatorname{Spec} R$. If $D$ is the closed point of $\operatorname{Spec} R$, then $C$ is a proper curve over the residue field $k$. Otherwise, $D$ is the closed subscheme of Spec $R$ defined by a height-1 prime ideal $\mathfrak{p} \subseteq R$. Since $R$ is two-dimensional and normal, the proper 
birational morphism $\mathscr{X} \rightarrow \operatorname{Spec} R$ is an isomorphism over codimension-1 points [Liu 2002, p. 150, Corollary 4.4.3]. Thus, the induced morphism $C \rightarrow D$ is proper, birational and quasifinite, and hence, by Chevalley's theorem, it is finite. Write $A=R / \mathfrak{p}$ so that $D=\operatorname{Spec} A$. Then $C=\operatorname{Spec} B$ for some domain $B \subseteq \kappa(C)=\kappa(D)$ that is finite over $A$. Since $A$ is a henselian excellent local domain, the same is true for $B$. The normalization $B^{\prime}$ of $B$ is finite over $B$ and hence a henselian local domain as well, and it coincides with the normalization of $A$ in its fraction field $\operatorname{Frac}(A)=\kappa(C)=\kappa(D)$. Then it is clear that $B^{\prime}$ is a henselian discrete valuation ring with residue field finite over $k$. This finishes the proof.

Recall that $\Omega_{R}$ denotes the set of discrete valuations of $K$ that are centered at codimension-1 points of regular proper models.

Corollary 3.16. For any $v \in \Omega_{R}$, the residue field $\kappa(v)$ is either the function field of a curve over $k$ or the fraction field of a henselian discrete valuation ring whose residue field is finite over $k$.

Now we can prove the following variant of [Saltman 2007, Corollary 5.2]:

Corollary 3.17. Let $q$ be a prime number unequal to the characteristic of the residue field $k$ and $\alpha \in \operatorname{Br}(K)[q]$ a Brauer class of order $q$. Let $\mathscr{\alpha} \rightarrow \operatorname{Spec} R$ be a regular proper model such that the ramification divisor $\operatorname{Ram}_{\mathscr{L}}(\alpha)$ of $\alpha$ on $\mathscr{X}$ has only simple normal crossings and that $\alpha$ has no cool points or chilly loops on $\mathscr{X}$. Write $\operatorname{ram}_{C_{i}}(\alpha)=\left(L_{i} / \kappa\left(C_{i}\right), \sigma_{i}\right)$ for the ramification data and $\beta_{i} \in \operatorname{Br}\left(\kappa\left(C_{i}\right)\right)$ for the residual classes.

Suppose that $k$ is a finite field. Then the following conditions are equivalent:

(i) $\alpha$ has index $q$,

(ii) $\beta_{i} \in \operatorname{Br}\left(\kappa\left(C_{i}\right)\right)$ is split by $L_{i} / \kappa\left(C_{i}\right)$ for every $i$ and

(iii) there are no hot points for $\alpha$ on $\mathscr{X}$.

Proof. Propositions 0.5 and 3.10(d) of [Saltman 2007] give (i) $\Longrightarrow$ (ii) $\Longrightarrow$ (iii).

To see (ii) $\Longrightarrow$ (i), note that by Proposition 3.11 and Remark 3.14 there is a degree- $q$ Kummer extension $M / K=K(\sqrt[q]{g}) / K$ that splits all the ramification of $\alpha$ over $R$. As the residue field is finite, we have $\operatorname{Br}_{\mathrm{nr}}(M / X)=0$ and in particular $\alpha_{M}=0$. Hence, the index of $\alpha$ divides $q$, the degree of the extension $M / K$. Since $\alpha$ has order $q$, it follows that the index of $\alpha$ is $q$.

To show (iii) $\Longrightarrow$ (ii), let $C$ be a fixed irreducible component of the ramification divisor $\operatorname{Ram}_{\mathscr{L}}(\alpha)$ with associated ramification data $\operatorname{ram}_{C}(\alpha)=(L / \kappa(C), \sigma)$. By [Saltman 2007, Lemma 4.1], there exists $\pi \in K^{*}$ having the following properties: (1) the valuation $s_{i}:=v_{C_{i}}(\pi)$ with respect to every component $C_{i}$ of $\operatorname{Ram}_{\mathscr{L}}(\alpha)$ is prime to $q$ and (2) whenever there is a chilly point $P$ in the intersection of two components $C_{i}$ and $C_{j}$, the coefficient of $P$ with respect to $C_{i}$ is equal to $s_{j} / s_{i}=v_{C_{j}}(\pi) / v_{C_{i}}(\pi) \bmod q$. 
Put $M:=K(\sqrt[q]{\pi})$. Let $\beta$ denote the residual class of $\alpha$ with respect to $M$, i.e., the specialization of $\alpha_{M} \in \operatorname{Br}(M)$ in $\operatorname{Br}(\kappa(C))$. We want to show that $\beta$ is split by $L / \kappa(C)$.

By Corollary 3.16, $\kappa(C)$ is either a function field in one variable over the finite field $k$ or the fraction field of a henselian discrete valuation ring with finite residue field. The same is true for $L$. So in either case, $\beta \in \operatorname{Br}(\kappa(C))[q]$ is split by $L / \kappa(C)$ if and only if $L / \kappa(C)$ splits all ramification of $\beta$ at every closed point $P$ of $C$.

Assume first that $L / \kappa(C)$ is split at $P$. Then $P$ is either a chilly point or a curve point ( $P$ is not cold because $L / \kappa(C)$ is unramified at $P$; see Definition 3.5). If $P$ is chilly, $\beta$ is unramified at $P$ by [Saltman 2007, Proposition 3.10(b)]. If $P$ is a curve point, then we conclude by [Saltman 2007, Proposition 3.11].

Next consider the case where $L / \kappa(C)$ is nonsplit at $P$. Then the $P$-adic valuation $v_{P}$ of $\kappa(C)$ extends uniquely to a discrete valuation $w_{P}$ of $L$. If $L / \kappa(C)$ is ramified at $P$, it is obvious that $L / \kappa(C)$ splits the ramification of $\beta$ at $P$. If $L / \kappa(C)$ is unramified at $P$, then $\kappa\left(w_{P}\right)$ is the unique degree- $q$ extension of the finite field $\kappa\left(v_{P}\right)=\kappa(P)$. Thus, the restriction map

$$
\text { Res : } H^{1}(\kappa(P), \mathbb{Z} / q \mathbb{Z}) \rightarrow H^{1}\left(\kappa\left(w_{P}\right), \mathbb{Z} / q \mathbb{Z}\right)
$$

is the zero map, which implies that $L / \kappa(C)$ splits the ramification of $\beta$ at $P$. The corollary is thus proved.

Example 3.18. Here is a concrete example that shows the bound on the periodindex exponent in Theorem 3.4 is sharp. The criterion in the above corollary will be used in the argument.

Let $p$ be a prime number such that $p \equiv 3(\bmod 4)$. Let $k=\mathbb{F}_{p}$ be the finite field of cardinality $p$, and let $R=k \llbracket x, y \rrbracket$ be the ring of formal power series in two variables $x$ and $y$ over $k$. Let $\mathscr{X} \rightarrow \operatorname{Spec} R$ be the blow-up of Spec $R$ at the closed point, and let $E \subseteq \mathscr{X}$ be the exceptional divisor. We have

$$
\operatorname{Proj}(k[T, S]) \cong E=\operatorname{Proj}(R[T, S] /(x, y)) \subseteq \mathscr{X}=\operatorname{Proj}(R[T, S] /(x S-y T)) .
$$

Let $f_{1}=y, f_{2}=x$ and $f_{3}=y+x$, and let $C_{i} \subseteq \mathscr{X}$ be the strict transform of the curve defined by $f_{i}=0$ in Spec $R$ for each $i=1,2,3$. Each intersection $C_{i} \cap E$ consists of a single point $P_{i}$.

Let $\alpha$ be the Brauer class of the biquaternion algebra $(-1, y) \otimes(y+x, x)$ over $K=k((x, y))$. The ramification divisor $\operatorname{Ram}_{\mathscr{L}}(\alpha)$ is $C_{1}+C_{2}+C_{3}+E$. The set of nodal points for $\alpha$ on $\mathscr{X}$ is $\left\{P_{1}, P_{2}, P_{3}\right\}$. Locally at $P_{1}$, we may choose $s$ and $x$ as local equations for $C_{1}$ and $E$, respectively, where $s=S / T=y / x \in K$. Thus, in the Brauer group $\operatorname{Br}(K)$, we have

$$
\alpha=(-1, y)+(y+x, x)=(-1, x s)+(x s+x, x)=(-1, s)+(s+1, x) .
$$


The function $s$ vanishes at $P_{1}$, and $-1 \neq 1 \in \kappa\left(P_{1}\right)^{*} / \kappa\left(P_{1}\right)^{* 2}=k^{*} / k^{* 2}$, so $P_{1}$ is a hot point by definition (Remark 3.6). One may verify that $P_{2}$ and $P_{3}$ are cold points.

As for the residual classes, one may check that for each $i$ the residual classes of $\alpha$ at $C_{i}$ are split by the ramification. Let us now show that at $E$ the residual classes are not split by the ramification. Indeed, if $v_{E}$ denotes the discrete valuation of $K$ defined by $E$, we have $v_{E}(x)=v_{E}(y)=v_{E}(y+x)=1$. Then it is easy to see that the ramification $\operatorname{ram}_{E}(\alpha)$ of $\alpha$ at $E$ is represented by the quadratic extension $k(s)(\sqrt{s+1})$ of $\kappa(E)=k(s)$. Putting $M=K(\sqrt{x}), \alpha_{M}=(-1, y)=$ $(-1, s) \in \operatorname{Br}(M)$, and hence, the residue class of $\alpha$ at $E$ with respect to $M / K$ is $\beta_{E}=(-1, s) \in \operatorname{Br}(\kappa(E))=\operatorname{Br}(k(s))$. Putting $u=\sqrt{s+1}$, it is easy to see that the quaternion algebra $(-1, s)=\left(-1, u^{2}-1\right)$ is not split over $k(u)=k(s)(\sqrt{s+1})$ (in fact, it ramifies at $u=1$ since -1 is not a square in $k=\mathbb{F}_{p}$ ).

By Theorem 3.4 and Corollary 3.17, we conclude that $\alpha \in \operatorname{Br}(K)[2]$ is of index 4 .

We shall now prove Theorem 1.3 in a slightly generalized form.

Theorem 3.19. Let $R$ be a two-dimensional, henselian, excellent local domain whose residue field $k$ has property $B_{1}, K$ the fraction field of $R, q$ a prime number unequal to the characteristic of $k$ and $\alpha \in \operatorname{Br}(K)[q]$. Assume either $\mu_{q} \subseteq R$ or $k$ is perfect.

If for every $v$ in the set $\Omega_{R}$ of discrete valuations of $K$ that correspond to codimension-1 points of regular proper models, the Brauer class $\alpha_{v}=\alpha \otimes_{K} K_{v} \in \operatorname{Br}\left(K_{v}\right)$ is represented by a cyclic algebra of degree $q$ over $K_{v}$, then $\alpha$ is represented by $a$ cyclic algebra of degree $q$ over $K$.

Proof. Let $\mathscr{X} \rightarrow \operatorname{Spec} R$ be a regular proper model such that $\operatorname{Ram}_{\mathscr{L}}(\alpha)$ is an snc divisor and that $\alpha$ has no cool points or chilly loops on $\mathscr{X}$. By Theorem 3.13 and Remark 3.14(2), it suffices to prove that all the residual classes of $\alpha$ at all components of $\operatorname{Ram}_{\mathscr{L}}(\alpha)$ are split by the ramification.

Assume the contrary. Then there is an irreducible component $C$ of $\operatorname{Ram}_{\mathscr{L}}(\alpha)$ such that the residual classes of $\alpha$ at $C$ are not split by the ramification. Now consider the discrete valuation $v=v_{C}$ of $K$ defined by $C$. By assumption, $\alpha_{v} \in \operatorname{Br}\left(K_{v}\right)[q]$ is cyclic of degree $q$ so that $\alpha_{v}=\left(\chi_{v}, b_{v}\right)$ for some $\chi_{v} \in H^{1}\left(K_{v}, \mathbb{Z} / q\right)$ and $b_{v} \in K_{v}^{*}$. Without loss of generality, we may assume $b_{v}=w \pi^{t}$, where $\pi \in K_{v}^{*}$ is a uniformizer for $v, w \in K_{v}^{*}$ is a unit for $v$ and $t$ is an integer such that $0 \leq t \leq q-1$.

Let $\left(L / K_{v}, \sigma_{v}\right)$ be the pair representing the character $\chi_{v} \in \operatorname{Hom}_{\mathrm{cts}}\left(G_{K_{v}}, \mathbb{Z} / q\right)$. If $L / K_{v}$ is unramified, then $t \neq 0$ because $\alpha$ is ramified at $v=v_{C}$. Then there exist integers $r, s \in \mathbb{Z}$ such that $1=r q+$ st. Putting $\pi^{\prime}=w^{s} \pi$, we have $b_{v}=w \pi^{t}=\left(w^{r}\right)^{q}\left(\pi^{\prime}\right)^{t}$. Then

$$
\alpha_{v}=\left(\chi_{v}, b_{v}\right)=\left(\chi_{v},\left(\pi^{\prime}\right)^{t}\right) \in \operatorname{Br}\left(K_{v}\right)[q]
$$

is clearly split by the totally ramified extension $K_{v}^{\prime} / K_{v}:=K_{v}\left(\sqrt[q]{\pi^{\prime}}\right) / K_{v}$. In particular, the residual class of $\alpha$ with respect to $K_{v}^{\prime} / K_{v}$ is 0 and a fortiori the 
residual classes of $\alpha$ at $C$ are split by the ramification. But this contradicts our choice of $C$.

If $L / K_{v}$ is ramified, then it is totally and tamely ramified. So $L=K_{v}(\sqrt[q]{\theta})$ for some $\theta \in K_{v}$. The extension being Galois, it follows that $\mu_{q} \subseteq L$. Since the residue fields of $L$ and $K_{v}$ are the same, Hensel's lemma implies that $\mu_{q} \subseteq K_{v}$. We may thus assume $\alpha_{v}=\left(u \pi^{s}, b_{v}\right)=\left(u \pi^{s}, w \pi^{t}\right)$ for some unit $u \in K_{v}^{*}$ and some integer $s$ such that $0 \leq s \leq q-1$. Since $\alpha$ is ramified at $v=v_{C}, s$ and $t$ cannot both be 0 . Assume for instance $s>0$. A similar argument as before shows that $\alpha_{v}$ is split by a totally ramified extension $K_{v}(\sqrt[q]{\pi}) / K_{v}$, which leads to a contradiction again. This proves the theorem.

The following special case, which answers a question in [Colliot-Thélène et al. 2002, Remark 3.7], will be used in Section 4:

Corollary 3.20. Let $R$ be a two-dimensional, henselian, excellent local domain with fraction field $K$ and residue field $k$. Assume that $k$ is a finite field of characteristic $\neq 2$. Let $D$ be a central simple algebra over $K$ of period 2 .

If for every $v \in \Omega_{R}, D \otimes_{K} K_{v}$ is Brauer equivalent to a quaternion algebra over $K_{v}$, then $D$ is Brauer equivalent to a quaternion algebra over $K$.

The analog of Theorem 3.19 in the case of a $p$-adic function field does not seem to have been noticed. Let us prove it in the following general form:

Theorem 3.21. Let $A$ be a henselian, excellent discrete valuation ring whose residue field $\kappa$ has property $B_{1}$. Let $q$ be a prime number unequal to the characteristic of $\kappa, F$ the function field of an algebraic curve over the fraction field of $A$ and $\alpha \in \operatorname{Br}(F)[q]$. For every regular surface 9 with function field $F$ equipped with a proper flat morphism $\mathrm{y} \rightarrow \operatorname{Spec} A$, let $\Omega\left(F / \mathrm{y}^{(1)}\right)$ denote the set of discrete valuations of $F$ corresponding to codimension-1 points of 9 . Let $\Omega_{A, F}$ be the union of all $\Omega\left(F / y^{(1)}\right)$, where y runs over regular surfaces as above.

Assume either $\mu_{q} \subseteq F$ or $\kappa$ is perfect. If, for every $v \in \Omega_{A, F}$,

$$
\alpha_{v}=\alpha \otimes_{F} F_{v} \in \operatorname{Br}\left(F_{v}\right)
$$

is represented by a cyclic algebra of degree $q$ over $F_{v}$, then $\alpha$ is represented by a cyclic algebra of degree $q$ over $F$.

Proof. By resolution of singularities, there exists a regular surface $X$ with function field $F$, together with a proper flat morphism $X \rightarrow \operatorname{Spec} A$, such that the ramification divisor $\operatorname{Ram}_{X}(\alpha)$ has only simple normal crossings and $\alpha$ has no cool points or chilly loops on $X$. By Corollary 3.12 and Remark 3.14, it suffices to show that all the residual classes of $\alpha$ are split by the ramification. This may be done as in the proof of Theorem 3.19. 


\section{Quadratic forms and the $u$-invariant}

A local-global principle from class field theory. To prove our results on quadratic forms, a result coming from Saito's work on class field theory for two-dimensional local rings will be used at a few crucial points.

As before, let $R$ be a two-dimensional, henselian, excellent local domain with fraction field $K$ and residue field $k$. The following proposition grows out of a conversation with S. Saito:

Proposition 4.1. Assume $R$ is normal and the residue field $k$ is finite. Suppose $\mathscr{L} \rightarrow \operatorname{Spec} R$ is a regular proper model such that the reduced divisor on the closed fiber has only simple normal crossings. Let $n>0$ be an integer that is invertible in $k$.

Then the natural map $H^{3}\left(K, \mu_{n}^{\otimes 2}\right) \rightarrow \prod_{v \in \mathscr{Q}(1)} H^{3}\left(K_{v}, \mu_{n}^{\otimes 2}\right)$. is injective.

Proof. Let $Y$ be the reduced subscheme on the closed fiber of $\mathscr{X} \rightarrow \operatorname{Spec} R$ and $U=\mathscr{X} \backslash Y$. Let $i: Y \rightarrow \mathscr{X}$ and $j: U \rightarrow \mathscr{X}$ denote the natural inclusions, and put $\mathscr{F}_{*}:=i^{*} R j_{*} \mu_{n}^{\otimes 2}$. Let $P=(\operatorname{Spec} R)^{(1)}$ be the set of codimension-1 points of Spec $R$. We may identify $P$ with the set of closed points of $U$ via the structural map $\mathscr{X} \rightarrow \operatorname{Spec} R$. From localization theories, we obtain exact sequences [Saito 1987, pp. 358-360]

$$
\begin{gathered}
H^{3}\left(U, \mu_{n}^{\otimes 2}\right) \stackrel{\phi}{\rightarrow} H^{3}\left(K, \mu_{n}^{\otimes 2}\right) \stackrel{\iota}{\rightarrow} \bigoplus_{\mathfrak{p} \in P} H^{3}\left(K_{\mathfrak{p}}, \mu_{n}^{\otimes 2}\right), \\
H^{3}(Y, \mathscr{F}) \rightarrow \bigoplus_{\eta \in Y^{(0)}} H^{3}(\kappa(\eta), \mathscr{F}) \stackrel{\theta^{\prime}}{\rightarrow} \bigoplus_{x \in Y^{(1)}} \mathbb{Z} / n,
\end{gathered}
$$

where the map $\iota$ in (4-1) induced by the natural maps $H^{3}\left(K, \mu_{n}^{\otimes 2}\right) \rightarrow H^{3}\left(K_{\mathfrak{p}}, \mu_{n}^{\otimes 2}\right)$. For each $\eta \in Y^{(0)} \subseteq \mathscr{X}^{(1)}$, let $A_{\eta}$ be the completion of the discrete valuation ring $\mathrm{O}_{\mathscr{X}, \eta}$. By the functoriality of the functor $R j_{*}$, we have the commutative diagram

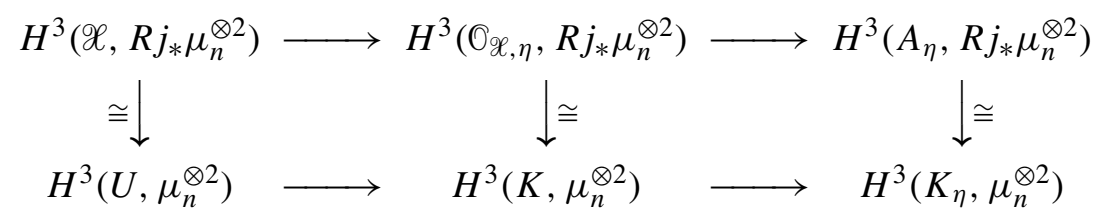

where the vertical maps are canonical isomorphisms. On the other hand, we have a commutative diagram

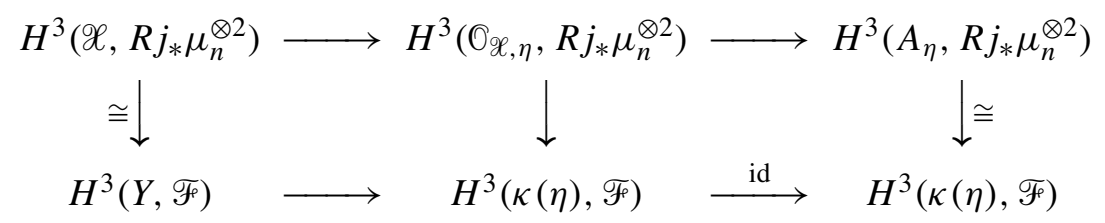


where the two vertical isomorphisms come from proper base change. Let

$$
\theta: \bigoplus_{\eta \in Y^{(0)}} H^{3}\left(K_{\eta}, \mu_{n}^{\otimes 2}\right) \rightarrow \bigoplus_{x \in Y^{(1)}} \mathbb{Z} / n
$$

be the composition of the map $\theta^{\prime}$ in (4-2) with the canonical isomorphism

$$
\bigoplus_{\eta \in Y^{(0)}} H^{3}\left(K_{\eta}, \mu_{n}^{\otimes 2}\right) \cong \bigoplus_{\eta \in Y^{(0)}} H^{3}(\kappa(\eta), \mathscr{F})
$$

Putting all things together, we get a commutative diagram with exact rows

$$
\begin{array}{cccc}
H^{3}\left(U, \mu_{n}^{\otimes 2}\right) & \stackrel{\phi}{\longrightarrow} H^{3}\left(K, \mu_{n}^{\otimes 2}\right) \stackrel{\iota}{\longrightarrow} \bigoplus_{\mathfrak{p} \in P} H^{3}\left(K_{\mathfrak{p}}, \mu_{n}^{\otimes 2}\right) \\
\cong \downarrow & \downarrow \downarrow & \\
H^{3}(Y, \mathscr{F}) & \stackrel{\tau}{\longrightarrow} \bigoplus_{\eta \in Y^{(0)}} H^{3}\left(K_{\eta}, \mu_{n}^{\otimes 2}\right) \stackrel{\theta}{\longrightarrow} \underset{x \in Y^{(1)}}{\bigoplus} \mathbb{Z} / n
\end{array}
$$

where the map $\varphi$ is induced by the restriction maps $H^{3}\left(K, \mu_{n}^{\otimes 2}\right) \rightarrow H^{3}\left(K_{\eta}, \mu_{n}^{\otimes 2}\right)$. By [Saito 1987, p. 361, Lemma 5.13], the induced map $\operatorname{Ker} \phi \rightarrow \operatorname{Ker} \tau$ is an isomorphism. Hence, $\varphi$ induces an isomorphism $\operatorname{Ker} \iota \cong \operatorname{Ker} \theta$. In particular, an element $\zeta \in H^{3}\left(K, \mu_{n}^{\otimes 2}\right)$ vanishes if and only if $\iota(\zeta)=0=\varphi(\zeta)$. The result then follows immediately since $\mathscr{X}^{(1)}=P \cup Y^{(0)}$.

An application of Proposition 4.1 that we will need is the following variant of [Parimala and Suresh 2010, Theorem 3.5] (see also [Parimala and Suresh 2012, Corollary 4.3]):

Theorem 4.2. Let $R$ be a two-dimensional, henselian, excellent local domain with finite residue field $k$ and fraction field $K$. Let $q$ be a prime number that is different from the characteristic of $k$. If $\mu_{q} \subseteq K$, then every element in $H^{3}\left(K, \mu_{q}^{\otimes 3}\right)$ is a symbol.

Proof. First note that as in [Parimala and Suresh 2010, Corollary 1.2], one can show that for any $v \in \Omega_{R}$, the kernel $H_{\mathrm{nr}}^{3}\left(K_{v}, \mu_{q}^{\otimes 3}\right)$ of the residue map

$$
H^{3}\left(K_{v}, \mu_{q}^{\otimes 3}\right) \stackrel{\partial_{v}}{\rightarrow} H^{2}\left(\kappa(v), \mu_{q}^{\otimes 2}\right)
$$

is trivial. Indeed, by Corollary 3.16, the residue field $\kappa(v)$ is either a function field in one variable over $k$ or the fraction field of a henselian discrete valuation ring $B$ whose residue field $k^{\prime}$ is finite over $k$. In the former case, we have $\operatorname{cd}_{q}(\kappa(v)) \leq 2$ by [Serre 1994, p. 93, Proposition 11] and hence $H^{3}\left(\kappa(v), \mu_{q}^{\otimes 3}\right)=0$. In the latter case, the exact sequence

$$
H^{3}\left(k^{\prime}, \mu_{q}^{\otimes 3}\right) \cong H_{\text {ét }}^{3}\left(B, \mu_{q}^{\otimes 3}\right) \rightarrow H^{3}\left(\kappa(v), \mu_{q}^{\otimes 3}\right) \rightarrow H^{2}\left(k^{\prime}, \mu_{q}^{\otimes 2}\right)
$$


implies $H^{3}\left(\kappa(v), \mu_{q}^{\otimes 3}\right)=0$ since $\operatorname{cd}\left(k^{\prime}\right) \leq 1$.

Let $\mathscr{X} \rightarrow$ Spec $R$ be a regular proper model. Since $H_{\mathrm{nr}}^{3}\left(K_{v}, \mu_{q}^{\otimes 3}\right)=0$ for all $v \in \Omega_{R}$, it follows that the kernel of the natural map

coincides with

$$
H^{3}\left(K, \mu_{q}^{\otimes 3}\right) \rightarrow \prod_{v \in \mathscr{\mathscr { C }}(1)} H^{3}\left(K_{v}, \mu_{q}^{\otimes 3}\right)
$$

$$
H_{\mathrm{nr}}^{3}\left(K / \mathscr{L}^{(1)}, \mu_{q}^{\otimes 3}\right):=\bigcap_{v \in \mathscr{C}^{(1)}} \operatorname{Ker}\left(\partial_{v}: H^{3}\left(K, \mu_{q}^{\otimes 3}\right) \rightarrow H^{2}\left(\kappa(v), \mu_{q}^{\otimes 2}\right)\right) .
$$

As $\mu_{q} \subseteq K$, it follows from Proposition 4.1 that $H_{\mathrm{nr}}^{3}\left(K / \mathscr{X}^{(1)}, \mu_{q}^{\otimes 3}\right)=0$. On the other hand, Lemma 3.15 implies that $\mathscr{X}$ is a $q$-special surface in the sense of [Parimala and Suresh 2012, §3]. So the result follows from [Parimala and Suresh 2012, Theorem 4.2].

Local-global principle for quadratic forms. Thanks to Corollary 3.20, we are now in a position to prove the local-global principle for quadratic forms of rank 5 with respect to the set $\Omega_{R}$ of divisorial valuations as asserted in Theorem 1.1. Standard notations from the algebraic theory of quadratic forms (as in [Lam 2005] or [Scharlau 1985]) will be used as of now.

Proof of Theorem 1.1. We follow the ideas in the proof of [Colliot-Thélène et al. 2002, Theorem 3.6]. Let $\phi$ be a five-dimensional nonsingular quadratic form over $K$. Assume that $\phi_{v}$ is isotropic over $K_{v}$ for every $v \in \Omega_{R}$.

The six-dimensional form $\psi:=\phi \perp\langle-\operatorname{det}(\phi)\rangle$ is similar to a so-called Albert form $\langle a, b,-a b,-c,-d, c d\rangle$. By the general theory of Albert forms [Gille and Szamuely 2006, p. 14, Theorem 1.5.5], the form $\langle a, b,-a b,-c,-d, c d\rangle$ is isotropic if and only if the biquaternion algebra $D:=(a, b) \otimes(c, d)$ is not a division algebra. By assumption, for every $v \in \Omega_{R}, \psi_{v}$ is isotropic over $K_{v}$, so the biquaternion algebra $D_{v}=(a, b)_{K_{v}} \otimes(c, d)_{K_{v}}$ is not a division algebra. The index of $D_{v}$ must be smaller than 4 , which is the degree. Therefore, $D_{v}$ is Brauer equivalent to a quaternion algebra over $K_{v}$. By Corollary 3.20, $D=(a, b) \otimes(c, d)$ is Brauer equivalent to a quaternion algebra over $K$. In particular, $D$ is not a division algebra over $K$. Hence, $\psi$ is isotropic over $K$. This implies that $\phi$ may be written in the form

$$
\phi=\operatorname{det}(\phi) .\langle 1, a, b, c, a b c\rangle
$$

over $K$. In particular, $\phi$ is similar to a subform of the threefold Pfister form

$$
\rho=\langle\langle-a,-b,-c\rangle\rangle:=\langle 1, a\rangle \otimes\langle 1, b\rangle \otimes\langle 1, c\rangle .
$$

By Merkurjev's theorem [Arason 1984, p. 129, Proposition 2], the form $\rho$ is isotropic if and only if the symbol class $(-a,-b,-c)$ vanishes. For each $v \in \Omega_{R}$, as the subform $\phi_{v}$ of $\rho_{v}$ is isotropic over $K_{v}$, we have $(-a,-b,-c)=0$ in $H^{3}\left(K_{v}, \mathbb{Z} / 2\right)$. 
Then it follows from Proposition 4.1 that $(-a,-b,-c)=0$ in $H^{3}(K, \mathbb{Z} / 2)$ (noticing that we may assume $R$ is normal). Thus, the Pfister form $\rho$ is isotropic over $K$ and hence hyperbolic [Lam 2005, p. 319, Theorem 1.7]. The form $\rho$ then contains a four-dimensional totally isotropic subspace, which must intersect the underlying space of the five-dimensional subform $\phi$ in a nontrivial subspace. Hence, $\phi$ is isotropic over $K$.

Remark 4.3. Let $R$ be a two-dimensional, henselian, excellent local domain with fraction field $K$ and residue field $k$. Assume the characteristic of $k$ is not 2. We record results and open questions on local-global principle for isotropy of quadratic forms over $K$ as far as we know.

(1) When the residue field $k$ is finite, Theorem 1.1 establishes the local-global principle with respect to discrete valuations in $\Omega_{R}$ only for rank-5 forms. If $R$ is of the form $R=A \llbracket t \rrbracket$, where $A$ is a complete discrete valuation ring, then the same local-global principle is proved for quadratic forms of any rank $\geq 5$ in $[\mathrm{Hu} 2012 \mathrm{~b}$, Theorem 1.2]. There the residue field may be any $C_{1}$-field.

(2) For general $R$ with finite residue field, it remains an open question whether the local-global principle holds for quadratic forms of rank 6,7 or 8 .

(3) Generalizing an earlier result of [Colliot-Thélène et al. 2002], [Hu 2012b, Theorem 1.1] proves the local-global principle for forms of rank 3 or 4 when the residue field $k$ is arbitrary (not necessarily finite, $C_{1}$ or $B_{1}$ ).

(4) The above results do not extend to binary forms even if $k$ is finite. For example, Jaworski [2001, Theorem 1.5] shows that if $K$ is the fraction field of the ring

$$
R=k \llbracket x, y, z \rrbracket /\left(z^{2}-\left(y^{2}-x^{3}\right)\left(x^{2}-y^{3}\right)\right),
$$

then $y^{2}-x^{3}$ is a square in $K_{v}$ for every discrete valuation $v$ of $K$, but it is not a square in $K$.

The u-invariant. Let $F$ be a field of characteristic $\neq 2$. Let $W(F)$ denote the Witt ring of quadratic forms over $F$, and $I(F)$ the fundamental ideal. For $a_{1}, \ldots, a_{n} \in F^{*}$, let $\left\langle\left\langle a_{1}, \ldots, a_{n}\right\rangle\right\rangle$ denote the $n$-fold Pfister form $\left\langle 1,-a_{1}\right\rangle \otimes \cdots \otimes\left\langle 1,-a_{n}\right\rangle$. The $n$-th power $I^{n}(F)$ of the fundamental ideal $I(F)$ is generated by the $n$-fold Pfister forms. Recall that the $u$-invariant of $F$, denoted $u(F)$, is defined as the supremum of dimensions of anisotropic quadratic forms over $F$ (so $u(F)=\infty$ if such dimensions can be arbitrarily large).

Let $R$ be a two-dimensional, henselian, excellent local domain with fraction field $K$ and residue field $k$. Assume that the residue field $k$ of $R$ is finite of characteristic $\neq 2$. Then the inequality $u(K) \geq 8$ may be easily seen as follows. Take any discrete valuation $v$ corresponding to a height-1 prime ideal of the normalization of $R$. It follows from Corollary 3.16 and a theorem of Springer [Scharlau 1985, 
p. 209, Corollary 2.6] that $u(\kappa(v))=4$. Take a four-dimensional diagonal form $\varphi$ over $K$ whose coefficients are units for $v$ such that its residue form $\bar{\varphi}$ over $\kappa(v)$ is anisotropic, and let $\pi \in K$ be a uniformizer for $v$. Then $\varphi \perp \pi . \varphi$ is an eightdimensional form over $K$ that is anisotropic over $K_{v}$.

The rest of this subsection is devoted to the proof of Theorem 1.2, which asserts that if $k$ is a finite field of characteristic $\neq 2$, then $u(K) \leq 8$ (or equivalently $u(K)=8$ according to the proceeding paragraph). Basically, we will follow the method of [Parimala and Suresh 2010] (see also [2012, Appendix]).

Proposition 4.4 [Parimala and Suresh 2010, Proposition 4.3]. Let $F$ be a field of characteristic $\neq 2$. Assume the following properties hold:

(i) $I^{4}(F)=0$.

(ii) Every element of $I^{3}(F)$ is represented by a threefold Pfister form.

(iii) Every element of $H^{3}(F, \mathbb{Z} / 2)$ is the sum of two symbols.

(iv) If $\phi$ is a threefold Pfister form and $\varphi_{2}$ is two-dimensional quadratic form over $F$, then there exist $f, a, b \in F^{*}$ such that $f$ is a value of $\varphi_{2}$ and $\phi=$ $\langle 1, f\rangle \otimes\langle 1, a\rangle \otimes\langle 1, b\rangle$ in the Witt group $W(F)$.

(v) If $\phi=\langle 1, f\rangle \otimes\langle 1, a\rangle \otimes\langle 1, b\rangle$ is a threefold Pfister form and $\varphi_{3}$ is threedimensional quadratic form over $F$, then there exist $g, h \in F^{*}$ such that $g$ is a value of $\varphi_{3}$ and $\phi=\langle 1, f\rangle \otimes\langle 1, g\rangle \otimes\langle 1, h\rangle$ in the Witt group $W(F)$.

Then $u(F) \leq 8$.

Property (i) in the above proposition is verified for the field $K$ by using the following deep, well-known theorem:

Theorem 4.5 (Artin-Gabber). Let $R$ be a two-dimensional, henselian, excellent local domain with fraction field $K$ and finite residue field $k$.

Then for every prime number $p$ different from the characteristic of $k$, the $p$ cohomological dimension $\mathrm{cd}_{p}(K)$ of $K$ is 3 .

When $K$ and $k$ have the same characteristic, this follows from a theorem of Artin [SGA 4.3 1973, Exposé XIX, Corollary 6.3]. When the characteristic of $K$ is different from that of $k$, Gabber proved the analog of Artin's result. A different proof due to Kato may be given along the lines of the case treated in [Saito 1986, $\S 5]$.

Corollary 4.6. Let $R$ be a two-dimensional, henselian, excellent local domain with finite residue field of characteristic $\neq 2$. Let $K$ be the fraction field of $R$.

Then $I^{4}(K)=0$.

Proof. This follows by combining Theorem 4.5 and a result of Arason, Elman and Jacob [Arason et al. 1986, Corollary 4]. 
Lemma 4.7. Let $R$ be a two-dimensional, henselian, excellent local domain with fraction field $K$ and residue field $k$. Assume that $k$ is a $B_{1}$ field of characteristic $\neq 2$. Then every element in $H^{2}(K, \mathbb{Z} / 2)$ is the sum of two symbols.

Proof. By Theorem 3.4, every element in $\operatorname{Br}(K)[2] \cong H^{2}(K, \mathbb{Z} / 2)$ has index dividing 4. A well-known theorem of Albert [1939, Chapter XI, §6, Theorem 9] then implies that it is the class of a tensor product of two quaternion algebras.

Proof of Theorem 1.2. We have $I^{3}(K) \cong H^{3}(K, \mathbb{Z} / 2)$ in view of Corollary 4.6 [Arason et al. 1986]. Thus, every element of $I^{3}(K)$ is represented by a threefold Pfister form by Theorem 4.2. That the field $K$ has property (iii) in Proposition 4.4 is proved in Lemma 4.7. Finally, the same argument as in the proof of [Parimala and Suresh 2012, Appendix, Proposition 3] proves that the field $K$ has properties (iv) and (v) in Proposition 4.4. The theorem is thus proved.

Question 4.8 (Suresh). Let $R, K$ and $k$ be as usual, and assume the residue field $k$ is an arbitrary (not necessarily finite) field of characteristic $\neq 2$. It is known that $u(K)=4 u(k)$ in each of the following special cases:

(1) $k$ is finite (Theorem 1.2).

(2) $k$ is hereditarily quadratically closed (i.e., every finite extension field of $k$ is quadratically closed). This basically follows from the proof of [Colliot-Thélène et al. 2002, Theorem 3.6].

(3) Assume that $u(L) \leq 2^{d} u(k)$ for every finitely generated field extension $L / k$ of transcendence degree $d \leq 1$. Then Harbater, Hartmann and Krashen have proved that $u(K)=4 u(k)$ holds in the following cases:

(a) $R=A \llbracket t \rrbracket$, where $A$ is a complete discrete valuation ring [Harbater et al. 2009, Corollary 4.19] and

(b) $K$ is a finite separable extension of $k((x, y))$ [Harbater et al. 2011, Corollary 4.2].

Question: Is the relation $u(K)=4 u(k)$ always true under the hypothesis of (3)? Is it true when assuming moreover $R$ is complete?

\section{Torsors under special orthogonal groups.}

Theorem 4.9. Let $R$ be a two-dimensional, henselian, excellent local domain with fraction field $K$ and residue field $k$. Assume $k$ is a finite field of characteristic $\neq 2$.

Then for any nonsingular quadratic form $\phi$ of rank $\geq 2$ over $K$, the natural map

$$
H^{1}(K, \mathrm{SO}(\phi)) \rightarrow \prod_{v \in \Omega_{R}} H^{1}\left(K_{v}, \mathrm{SO}(\phi)\right)
$$

is injective. 
Proof. Let $\psi$ and $\psi^{\prime}$ be nonsingular quadratic forms representing classes in $H^{1}(K, \mathrm{SO}(\phi))$. As they have the same dimension, the forms $\psi$ and $\psi^{\prime}$ are isometric if and only if they represent the same class in the Witt group. Since $\psi$ and $\psi^{\prime}$ also have the same discriminant, it follows from [Scharlau 1985, p. 82, Chapter 2, Lemma 12.10] that $\psi-\psi^{\prime} \in I^{2}(K)$. Now it suffices to apply Lemma 4.10 below.

Lemma 4.10. Let $R, K$ and $k$ be as in Theorem 4.9. The natural map

is injective.

$$
I^{2}(K) \rightarrow \prod_{v \in \Omega_{R}} I^{2}\left(K_{v}\right)
$$

Proof. In the case where $R$ is the henselization of an algebraic surface over a finite field at a closed point, this is already established in [Colliot-Thélène et al. 2002, Theorem 3.10]. Here the argument is essentially the same with Proposition 4.1 and Corollary 4.6 substituting appropriate ingredients in that case.

Remark 4.11. In Theorem 4.9, if $\phi$ is of dimension 2 or 3, one need not assume the residue field $k$ finite.

Indeed, let $\psi$ and $\psi^{\prime}$ be nonsingular forms representing classes in $H^{1}(K, \mathrm{SO}(\phi))$. In the two-dimensional case, assume $\psi^{\prime} \cong\langle a, b\rangle$ and $\psi \cong\langle\alpha, \beta\rangle$. Then $\psi^{\prime} \cong \psi$ if and only if the quaternion algebras $(a, b)$ and $(\alpha, \beta)$ are isomorphic since the two forms have the same discriminant [Scharlau 1985, Chapter 2, Corollary 11.11]. In the three-dimensional case, assume $\psi^{\prime} \cong\langle a, b, c\rangle$ and $\psi \cong\langle\alpha, \beta, \gamma\rangle$. Then $\psi^{\prime} \cong \psi$ if and only if the quaternion algebras $(-a c,-b c)$ and $(-\alpha \gamma,-\beta \gamma)$ are isomorphic [Scharlau 1985, Chapter 2, Theorem 11.12]. Since two quaternion algebras are isomorphic if and only if their classes in the Brauer group coincide, the result then follows from the injectivity of the natural map

$$
\operatorname{Br}(K) \rightarrow \prod_{v \in \Omega_{R}} \operatorname{Br}\left(K_{v}\right),
$$

this last local-global statement being essentially established in [Colliot-Thélène et al. 2002, §1] (see also the proof of [Hu 2012b, Theorem 1.1]).

Remark 4.12. Let $F$ be a field of characteristic $\neq 2$ and $\Omega$ a set of discrete valuations of $F$. For each integer $r \geq 2$, consider the following statements:

$\left(\mathrm{LG}_{r}\right)$ For any two nonsingular quadratic forms of rank $r$ that have the same discriminant over $F$, if they are isometric over $F_{v}$ for every $v \in \Omega$, then they must already be isometric over $F$.

$\left(\mathrm{LG}_{r}^{\prime}\right)$ If a nonsingular quadratic form of rank $r$ over $F$ is isotropic over $F_{v}$ for every $v \in \Omega$, then it is isotropic over $F$.

Theorem 4.9 amounts to saying that for every $r \geq 2,\left(\mathrm{LG}_{r}\right)$ is true for the field $K$ with respect to its divisorial valuations. Our proof of this theorem does not rely on 
the local-global principle for the isotropy of quadratic forms. Note however that over an arbitrary field $F$ (of characteristic $\neq 2$ ) one has $\left(\mathrm{LG}_{r}\right)+\left(\mathrm{LG}_{r+2}^{\prime}\right) \Longrightarrow\left(\mathrm{LG}_{r+1}\right)$.

Indeed, let $\psi, \psi^{\prime}$ be nonsingular quadratic forms of rank $r+1$ over $F$ that have the same discriminant. Assume $\psi \cong\left\langle a_{1}\right\rangle \perp \psi_{1}$ with $\psi_{1}$ of rank $r$. If $(\psi)_{F_{v}} \cong\left(\psi^{\prime}\right)_{F_{v}}$ for every $v \in \Omega$, then $\left(\psi^{\prime} \perp\left\langle-a_{1}\right\rangle\right)_{F_{v}}$ is isotropic for every $v \in \Omega$. By the local-global principle $\left(\mathrm{LG}_{r+2}^{\prime}\right), \psi^{\prime}$ represents $a_{1}$ over $F$ whence a decomposition $\psi^{\prime} \cong\left\langle a_{1}\right\rangle \perp \psi_{1}^{\prime}$. It then suffices to apply $\left(\mathrm{LG}_{r}\right)$ to the forms $\psi_{1}$ and $\psi_{1}^{\prime}$, thanks to Witt's cancellation theorem.

Together with the argument in Remark 4.11, this shows that if the natural map $\operatorname{Br}(F) \rightarrow \prod_{v \in \Omega} \operatorname{Br}\left(F_{v}\right)$ is injective and if the local-global principle with respect to $\Omega$ holds for quadratic forms of rank $\geq 5$ over $F$, then $\left(\mathrm{LG}_{r}\right)$ is true for all $r \geq 2$. In particular, if $F$ is the function field of an algebraic curve over the fraction field of a complete discrete valuation ring with arbitrary residue field of characteristic $\neq 2$, then the analog of Theorem 4.9 over $F$ is true by [Colliot-Thélène et al. 2012, Theorems 3.1 and 4.3]. Note also that in this situation $\left(\mathrm{LG}_{2}^{\prime}\right)$ is false in general.

\section{Acknowledgements}

I got interested in the problems considered in this paper when I was attending the workshop "Deformation theory, patching, quadratic forms, and the Brauer group" held at American Institute of Mathematics in Palo Alto, CA, in January 2011. I thank AIM and the organizers of this workshop for their kind hospitality and generous support. I'm grateful to my advisor, Professor Jean-Louis Colliot-Thélène, for helpful discussions and comments. Thanks are also due to Professor Shuji Saito, with whom a conversation has helped me find the answer to a question that is needed in the paper. I also thank Professors Raman Parimala and Venapally Suresh for sending me their new preprint on degree-3 cohomology. I'm indebted to the referee for a long list of valuable comments.

\section{References}

[Albert 1939] A. A. Albert, Structure of algebras, Amer. Math. Soc. Colloq. Publ. 24, American Mathematical Society, Providence, RI, 1939. Reprinted 1961. MR 1,99c Zbl 0023.19901

[Arason 1984] J. K. Arason, “A proof of Merkurjev's theorem”, pp. 121-130 in Quadratic and Hermitian forms (Hamilton, ON, 1983), CMS Conf. Proc. 4, American Mathematical Society, Providence, RI, 1984. MR 86f:11029 Zbl 0556.10009

[Arason et al. 1986] J. K. Arason, R. Elman, and B. Jacob, "Fields of cohomological 2-dimension three", Math. Ann. 274:4 (1986), 649-657. MR 87m:12006 Zbl 0576.12025

[Brussel 2010] E. Brussel, "On Saltman's p-adic curves papers", pp. 13-39 in Quadratic forms, linear algebraic groups, and cohomology, edited by J.-L. Colliot-Thélène et al., Dev. Math. 18, Springer, New York, 2010. MR 2011k:16041 Zbl 1245.16014

[Colliot-Thélène 1995] J.-L. Colliot-Thélène, "Birational invariants, purity and the Gersten conjecture", pp. 1-64 in $K$-theory and algebraic geometry: connections with quadratic forms and division 
algebras (Santa Barbara, CA, 1992), edited by B. Jacob and A. Rosenberg, Proc. Sympos. Pure Math. 58, American Mathematical Society, Providence, RI, 1995. MR 96c:14016 Zbl 0834.14009

[Colliot-Thélène 1998] J.-L. Colliot-Thélène, “Zentralblatt MATH review of [Saltman 1997]”, 1998, Available at http://www.zentralblatt-math.org/zmath/en/advanced/?q=an:0902.16021.

[Colliot-Thélène 2006] J.-L. Colliot-Thélène, "Algèbres simples centrales sur les corps de fonctions de deux variables (d'après A. J. de Jong) (Exposé 949)", pp. 379-413 in Séminaire Bourbaki 2004/2005, Astérisque 307, Société Mathématique de France, Paris, 2006. MR 2008b:14078 Zbl 1123.14012

[Colliot-Thélène et al. 2002] J.-L. Colliot-Thélène, M. Ojanguren, and R. Parimala, "Quadratic forms over fraction fields of two-dimensional Henselian rings and Brauer groups of related schemes", pp. 185-217 in Algebra, arithmetic and geometry (Mumbai, 2000), vol. 1, edited by R. Parimala, Tata Inst. Fund. Res. Stud. Math. 16, Narosa Publishing House, New Delhi, 2002. MR 2004c:14031 Zbl 1055.14019

[Colliot-Thélène et al. 2012] J.-L. Colliot-Thélène, R. Parimala, and V. Suresh, "Patching and localglobal principles for homogeneous spaces over function fields of p-adic curves", Comment. Math. Helv. 87:4 (2012), 1011-1033. MR 2984579 Zbl 06104833

[Gille and Szamuely 2006] P. Gille and T. Szamuely, Central simple algebras and Galois cohomology, Cambridge Studies in Advanced Mathematics 101, Cambridge University Press, 2006. MR 2007k:16033 Zbl 1137.12001

[Grothendieck 1961] A. Grothendieck, "Éléments de géométrie algébrique, III: Étude cohomologique des faisceaux cohérents,I", Inst. Hautes Études Sci. Publ. Math. 11 (1961), 5-167. MR 36 \#177a Zbl 0118.36206

[Grothendieck 1967] A. Grothendieck, "Éléments de géométrie algébrique, IV: Étude locale des schémas et des morphismes de schémas, IV", Inst. Hautes Études Sci. Publ. Math. 32 (1967), 5-361. MR 39 \#220 Zbl 0153.22301

[Grothendieck 1968a] A. Grothendieck, "Le groupe de Brauer, II: Théorie cohomologique", pp. 67-87 in Dix exposés sur la cohomologie des schémas, North-Holland, Amsterdam, 1968. MR 39 \#5586b Zbl 0198.25803

[Grothendieck 1968b] A. Grothendieck, "Le groupe de Brauer, III: Exemples et compléments", pp. 88-188 in Dix exposés sur la cohomologie des schémas, North-Holland, Amsterdam, 1968. MR 39 \#5586c Zbl 0198.25901

[Harbater et al. 2009] D. Harbater, J. Hartmann, and D. Krashen, "Applications of patching to quadratic forms and central simple algebras”, Invent. Math. 178:2 (2009), 231-263. MR 2010j:11058 Zbl 05627032

[Harbater et al. 2011] D. Harbater, J. Hartmann, and D. Krashen, "Weierstrass preparation and algebraic invariants", preprint, 2011. arXiv 1109.6362

[Heath-Brown 2010] D. R. Heath-Brown, "Zeros of systems of p-adic quadratic forms", Compos. Math. 146:2 (2010), 271-287. MR 2011e:11066 Zbl 1194.11047

[Hoffmann and Van Geel 1998] D. W. Hoffmann and J. Van Geel, "Zeros and norm groups of quadratic forms over function fields in one variable over a local non-dyadic field", J. Ramanujan Math. Soc. 13:2 (1998), 85-110. MR 2000c:11058 Zbl 0922.11032

[Hu 2012a] Y. Hu, "Hasse principle for simply connected groups over function fields of surfaces", preprint, 2012. arXiv 1203.1075

[Hu 2012b] Y. Hu, "Local-global principle for quadratic forms over fraction fields of two-dimensional Henselian domains", Ann. Inst. Fourier (Grenoble) 62:6 (2012), 2131-2143 (2013). MR 3060754 Zbl 06159908 
[Jaworski 2001] P. Jaworski, "On the strong Hasse principle for fields of quotients of power series rings in two variables”, Math. Z. 236:3 (2001), 531-566. MR 2002h:11034 Zbl 1009.11027

[de Jong 2004] A. J. de Jong, "The period-index problem for the Brauer group of an algebraic surface”, Duke Math. J. 123:1 (2004), 71-94. MR 2005e:14025 Zbl 1060.14025

[Kato 1986] K. Kato, "A Hasse principle for two-dimensional global fields", J. Reine Angew. Math. 366 (1986), 142-183. MR 88b:11036 Zbl 0576.12012

[Lam 2005] T. Y. Lam, Introduction to quadratic forms over fields, Graduate Studies in Mathematics 67, American Mathematical Society, Providence, RI, 2005. MR 2005h:11075 Zbl 1068.11023

[Leep 2013] D. B. Leep, “The $u$-invariant of $p$-adic function fields”, preprint, 2013.

[Lieblich 2011a] M. Lieblich, "Period and index in the Brauer group of an arithmetic surface", $J$. Reine Angew. Math. 659 (2011), 1-41. MR 2837009 Zbl 1230.14021

[Lieblich 2011b] M. Lieblich, “The period-index problem for fields of transcendence degree 2", preprint, 2011. arXiv 0909.4345v2

[Lipman 1975] J. Lipman, "Introduction to resolution of singularities", pp. 187-230 in Algebraic geometry (Arcata, CA, 1974), edited by R. Hartshorne, Proc. Sympos. Pure Math. 29, American Mathematical Society, Providence, RI, 1975. MR 52 \#10730 Zbl 0306.14007

[Lipman 1978] J. Lipman, "Desingularization of two-dimensional schemes", Ann. Math. (2) 107:1 (1978), 151-207. MR 58 \#10924 Zbl 0349.14004

[Liu 2002] Q. Liu, Algebraic geometry and arithmetic curves, Oxford Graduate Texts in Mathematics 6, Oxford University Press, 2002. MR 2003g:14001 Zbl 0996.14005

[Milne 1980] J. S. Milne, Étale cohomology, Princeton Mathematical Series 33, Princeton University Press, 1980. MR 81j:14002 Zbl 0433.14012

[Parimala and Suresh 2010] R. Parimala and V. Suresh, "The $u$-invariant of the function fields of p-adic curves", Ann. of Math. (2) 172:2 (2010), 1391-1405. MR 2011g:11074 Zbl 1208.11053

[Parimala and Suresh 2012] R. Parimala and V. Suresh, "Degree three cohomology of function fields of surfaces", preprint, 2012.

[Saito 1986] S. Saito, "Arithmetic on two-dimensional local rings", Invent. Math. 85:2 (1986), 379-414. MR 87j:11060 Zbl 0609.13003

[Saito 1987] S. Saito, "Class field theory for two-dimensional local rings", pp. 343-373 in Galois representations and arithmetic algebraic geometry (Kyoto, 1985, Tokyo, 1986), edited by Y. Ihara, Adv. Stud. Pure Math. 12, North-Holland, Amsterdam, 1987. MR 90h:11053 Zbl 0672.12006

[Saltman 1997] D. J. Saltman, "Division algebras over $p$-adic curves", J. Ramanujan Math. Soc. 12:1 (1997), 25-47. Correction in 13:2 (1998), 125-129. MR 98d:16032 Zbl 0902.16021

[Saltman 1998] D. J. Saltman, “Correction to 'Division algebras over p-adic curves' (J. Ramanujan Math. Soc. 12:1 (1997), 25-47)”, J. Ramanujan Math. Soc. 13:2 (1998), 125-129. MR 99k:16036

[Saltman 2007] D. J. Saltman, “Cyclic algebras over p-adic curves”, J. Algebra 314:2 (2007), 817843. MR 2008i:16018 Zbl 1129.16014

[Saltman 2008] D. J. Saltman, "Division algebras over surfaces”, J. Algebra 320:4 (2008), 1543-1585. MR 2009d:16028 Zbl 1171.16011

[Scharlau 1985] W. Scharlau, Quadratic and Hermitian forms, Grundlehren Math. Wiss. 270, Springer, Berlin, 1985. MR 86k:11022 Zbl 0584.10010

[Serre 1994] J.-P. Serre, Cohomologie galoisienne, 5th ed., Lecture Notes in Mathematics 5, Springer, Berlin, 1994. MR 96b:12010 Zbl 0812.12002 
[SGA 4.2 1972] M. Artin, A. Grothendieck, and J. L. Verdier, Séminaire de Géométrie Algébrique du Bois Marie 1963/64: Théorie des topos et cohomologie étale des schémas (SGA 4), tome 2, Lecture Notes in Mathematics 270, Springer, Berlin, 1972. MR 50 \#7131 Zbl 0237.00012

[SGA 4.3 1973] M. Artin, A. Grothendieck, and J. L. Verdier, Séminaire de Géométrie Algébrique du Bois Marie 1963/64: Théorie des topos et cohomologie étale des schémas (SGA 4), tome 3, Lecture Notes in Mathematics 305, Springer, Berlin, 1973. MR 50 \#7132 Zbl 0245.00002

[Shafarevich 1966] I. R. Shafarevich, Lectures on minimal models and birational transformations of two dimensional schemes, Tata Inst. Fund. Res. Lectures on Math. and Phys. 37, Tata Institute of Fundamental Research, Bombay, 1966. MR 36 \#163 Zbl 0164.51704

Communicated by Raman Parimala

Received 2012-05-31 Revised 2012-09-09 Accepted 2012-10-15

hu1983yong@gmail.com Université Paris-Sud 11, 15 rue Georges Clemenceau, Mathématiques, Bâtiment 425, 91405 Orsay Cedex, France 


\section{Algebra \& Number Theory}

msp.org/ant

\section{EDITORS}

MANAGING EDITOR

Bjorn Poonen

Massachusetts Institute of Technology

Cambridge, USA

\author{
EDITORIAL BOARD CHAIR \\ David Eisenbud \\ University of California \\ Berkeley, USA
}

\section{BOARD OF EDITORS}

Georgia Benkart

Dave Benson

Richard E. Borcherds

John H. Coates

J-L. Colliot-Thélène

Brian D. Conrad

Hélène Esnault

Hubert Flenner

Edward Frenkel

Andrew Granville

Joseph Gubeladze

Roger Heath-Brown

Ehud Hrushovski

Craig Huneke

Mikhail Kapranov

Yujiro Kawamata

János Kollár

Yuri Manin

Barry Mazur

Philippe Michel
University of Wisconsin, Madison, USA

University of Aberdeen, Scotland

University of California, Berkeley, USA

University of Cambridge, UK

CNRS, Université Paris-Sud, France

University of Michigan, USA

Freie Universität Berlin, Germany

Ruhr-Universität, Germany

University of California, Berkeley, USA

Université de Montréal, Canada

San Francisco State University, USA

Oxford University, UK

Hebrew University, Israel

University of Virginia, USA

Yale University, USA

University of Tokyo, Japan

Princeton University, USA

Northwestern University, USA

Harvard University, USA

École Polytechnique Fédérale de Lausanne
Susan Montgomery

Shigefumi Mori

Raman Parimala

Jonathan Pila

Victor Reiner

Karl Rubin

Peter Sarnak

Joseph H. Silverman

Michael Singer

Vasudevan Srinivas

J. Toby Stafford

Bernd Sturmfels

Richard Taylor

Ravi Vakil

Michel van den Bergh

Marie-France Vignéras

Kei-Ichi Watanabe

Efim Zelmanov

Shou-Wu Zhang
University of Southern California, USA

RIMS, Kyoto University, Japan

Emory University, USA

University of Oxford, UK

University of Minnesota, USA

University of California, Irvine, USA

Princeton University, USA

Brown University, USA

North Carolina State University, USA

Tata Inst. of Fund. Research, India

University of Michigan, USA

University of California, Berkeley, USA

Harvard University, USA

Stanford University, USA

Hasselt University, Belgium

Université Paris VII, France

Nihon University, Japan

University of California, San Diego, USA

Princeton University, USA

PRODUCTION

production@msp.org

Silvio Levy, Scientific Editor

See inside back cover or msp.org/ant for submission instructions.

The subscription price for 2013 is US $\$ 200 /$ year for the electronic version, and $\$ 350 /$ year $(+\$ 40$, if shipping outside the US) for print and electronic. Subscriptions, requests for back issues and changes of subscribers address should be sent to MSP.

Algebra \& Number Theory (ISSN 1944-7833 electronic, 1937-0652 printed) at Mathematical Sciences Publishers, 798 Evans Hall \#3840, c/o University of California, Berkeley, CA 94720-3840 is published continuously online. Periodical rate postage paid at Berkeley, CA 94704, and additional mailing offices.

ANT peer review and production are managed by EditFLOW ${ }^{\circledR}$ from Mathematical Sciences Publishers.

\section{PUBLISHED BY}

- mathematical sciences publishers

nonprofit scientific publishing

http://msp.org/

(C) 2013 Mathematical Sciences Publishers 


\section{Algebra \& Number Theory}

Volume $7 \quad$ No. $8 \quad 2013$

The geometry and combinatorics of cographic toric face rings

1781

Sebastian Cas alaina-Martin, Jesse Leo Kass and Filippo Viviani

Essential $p$-dimension of algebraic groups whose connected component is a torus

Roland LÖTscher, MARK MacDonald, AUREl MEYer and Zinovy

REICHSTEIN

Differential characterization of Wilson primes for $\mathbb{F}_{q}[t]$

DINESH S. THAKUR

Principal $W$-algebras for $\mathrm{GL}(m \mid n)$

JONATHAN BROWN, JONATHAN BRUNDAN and SimON M. GOODWIN

Kernels for products of $L$-functions

NiKOLAOS Diamantis and CORMAC O'SUllivaN

Division algebras and quadratic forms over fraction fields of two-dimensional henselian domains

YONG HU

The operad structure of admissible $G$-covers

DAN PETERSEN

The $p$-adic monodromy theorem in the imperfect residue field case

SHUN OHKUBO

On the Manin-Mumford and Mordell-Lang conjectures in positive characteristic

DAMIAN RÖSSLER 